\title{
siRNA Silencing by Chemically Modified Biopolymeric Nanovectors
}

Eva Villar-Alvarez, ${ }^{*},{ }^{\dagger}$, Baltazar H. Leal ${ }^{\dagger, \neq, \S}$ Raquel Martínez-González, ${ }^{\dagger}$ Alberto Pardo, ${ }^{\dagger}$ Sonia Al-Qadi, ${ }^{\dagger}$ Josué Juárez, ${ }^{\ddagger}$ Miguel A. Váldez, ${ }^{*}$ Adriana Cambón, ${ }^{\dagger}$ Silvia Barbosa, ${ }^{* \dagger \odot}$ and Pablo Taboada ${ }^{\dagger \odot}$

${ }^{\dagger}$ Colloids and Polymers Physics Group, Particle Physics Department, Universidade de Santiago de Compostela, 15782 Santiago de Compostela, Spain

${ }^{\ddagger}$ Departamento de Física, Universidad de Sonora, Rosales y Transversal, 83000 Hermosillo Sonora, Mexico

\section{Supporting Information}

ABSTRACT: The ability to specifically silence genes by RNA interference has an enormous potential for treating genetic diseases. However, different drawbacks such as short interfering RNA (siRNA) degradability by serum nucleases and biodistribution issues still need to be overcome to develop suitable delivery vehicles that have been proven essential in carrying siRNA to its target. Chitosan is an attractive biomaterial to construct gene nanocarriers as it is safe, cheap, and amenable to chemical modifications. However, the transfection efficiency of nanovectors based on unmodified
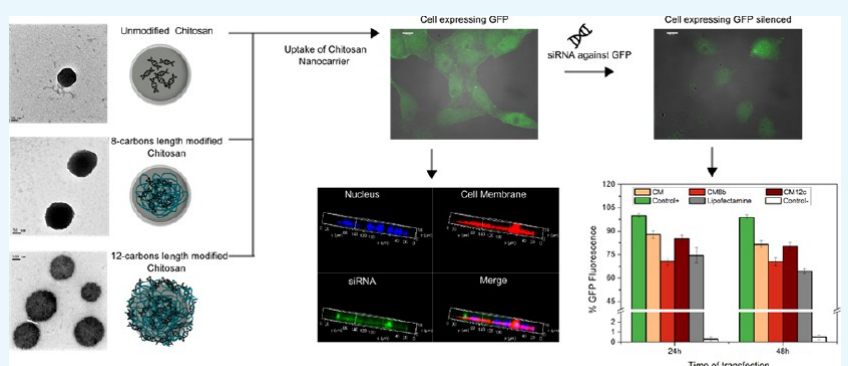
chitosan has revealed to be relatively low and dependent on different factors such as the biopolymer molecular weight, deacetylation degree, charge ratio, $\mathrm{pH}$, or particle size. Thus, specific strategies have been adopted to improve the transfection efficacy of chitosan-based nanovectors. In this work, hydrophobically modified chitosans with 8-, 10-, and 12-carbon side chains grafted to the polymeric backbone by a reductive amination process were used to develop polymeric nanoparticles by the ionotropic gelation method. After chitosan modification, the produced nanoparticles showed a suitable combination of size and surface charge with high siRNA loading capacities, efficient protection against serum nucleases, and satisfactory in vitro release profiles. Importantly, the introduced structural modifications were observed to modulate the overall physicochemical characteristics of the nanoparticles including their biological performance like their cell viability, uptake, and transfection efficiency. In this regard, the knockdown activity of the prepared nanoparticles was tested in HeLa cells overexpressing the green fluorescent protein after 24 and $48 \mathrm{~h}$ of incubation, observing a silencing activity greater than that displayed by the commercial transfection agent Lipofectamine 2000.

\section{INTRODUCTION}

In comparison to conventional DNA-based gene therapy, the use of short interfering RNAs (siRNAs) for silencing genes through RNA interference (RNAi) has recently attracted much attention. ${ }^{1}$ This is a biological process in which siRNA molecules interfere in the expression of specific genes by degrading targeted messenger RNA (mRNA) after transcription; this prevents their translation and, subsequently, leads to gene silencing. Upon introduction into cells, siRNA joins a multicomponent nuclease complex termed RNAinduced silencing complex (RISC). Within the RISC, siRNA is unrolled to form single-stranded siRNA, where one strand is integrated as a template within the RISC complex for recognizing complementary mRNA strands. After strand targeting, ribonucleases are activated inducing mRNA strand cleavage and subsequent suppression of gene/protein expression associated with mRNA codification. ${ }^{2,3}$ The nonincorporated mRNA strand is eliminated during the siRNA assembly process and, probably, degraded thereafter. ${ }^{4}$ RNAi is advantageous over other therapies in terms of, for example, its specificity and noncytotoxicity. ${ }^{3}$ In addition, the effective administration of synthetic siRNAs in vivo and the subsequent downregulation of an endogenous target without undesirable side effects have been already demonstrated. ${ }^{5}$ Thanks to their selectivity and potency, RNAi is becoming a very attractive approach for future therapeutics to provide specific gene expression silencing and subsequent control of gene disorders.

Nevertheless, for an effective action and potential widespread use of siRNA therapeutics, several major challenges must be first overcome: (i) the achievement of an optimal concentration of the oligonucleotide double strands inside the cell cytoplasms to induce effective and long-standing gene silencing; (ii) the protection of siRNA from degradation by serum nucleases after administration leading to enhanced halflives; $^{6}$ (iii) the impossibility of siRNA strands to diffuse through cellular membranes by their anionic character and size, the former involving strong repulsive electrostatic interactions with cell membranes. ${ }^{7}$ Therefore, the efficient, safe, and repeated administration of siRNAs is largely dependent on the development of suitable nanocarriers for their controlled

Received: October 19, 2018

Accepted: February 12, 2019

Published: February 21, 2019 
transport and delivery. ${ }^{8}$ An ideal carrier for siRNA transport and delivery should, then, fulfill the following requirements: (i) to be able to bind and condense siRNA strands inside the nanovehicle; (ii) to protect this therapeutic cargo from serumdegrading enzymes; (iii) to target the therapeutics specifically to the site of action; (iv) to facilitate/enhance cellular uptake and internalization of the bioactive material; (v) to allow the escape from endosomes/lysosomes into the cytosol; and finally, (vi) to promote effective silencing and subsequent downregulation.

Previous studies have shown chitosan as a very promising carrier biomaterial for RNA delivery to attain an effective expression of reporter genes in vitro and in vivo, highlighting the potential use of chitosan as the main constituent of nanocarriers for siRNA delivery. To establish its effectiveness, it is key to determine the influence of the formulation parameters on the physical and biological performances of the formed siRNA/chitosan complexes for an effective therapeutic response and to ensure correct siRNA protection to enzymatic degradation. For example, Liu et al. analyzed the in vitro gene silencing of chitosan-siRNA nanoparticles (CSNPs), elucidating that this phenomenon is strongly dependent on the molecular weight $\left(M_{\mathrm{w}}\right)$ and degree of deacetylation of the biopolymer. ${ }^{9}$ Katas et al. showed that the transfection efficiency of siRNA depends on the method of siRNA association with chitosan, with ionic gelation being the best methodology to provide the highest biological effect. ${ }^{10} \mathrm{~A}$ remarkable in vivo study developed by Howard et al. also demonstrated the efficacy of nasally administered siRNAencapsulated chitosan NPs in transgenic mice overexpressing the green fluorescent protein (GFP), in which significant GFP knockdown was achieved. ${ }^{11}$ Recently, additional studies have further investigated the role played by the biopolymer hydrophobicity, nitrogen-to-phosphate $(\mathrm{N} / \mathrm{P})$ ratio, particle size, and targeting ligands in the improvement of the transfection efficiency and associated gene knockdown. For instance, the combination of pRNA dimers with poly(ethylene glycol) (PEG)-modified chitosan NPs functionalized with folate gave rise to a tumor-targeting siRNA delivery system with a much higher cargo accumulation in the in vivo target tissue. $^{12}$

However, chitosan-based siRNA delivery nanocarriers have their own limitations. One of the main drawbacks is their low water solubility at physiological $\mathrm{pH}$, which may preclude their successful in vivo administration. It is worth reminding that at physiological $\mathrm{pH}$, the number of available positively charged groups in the chitosan backbone is largely reduced, which can impede the siRNA electrostatic complexation and preclude particle stability in vivo. Moreover, the transfection efficiency of native chitosan is still far to be optimal, and insufficient in vivo siRNA intracellular release levels have been achieved. ${ }^{13}$ This major issue may originate from serum proteins (albumin and fibrinogen), which can cause particle aggregation, favoring the probability of suffering embolism and/or enhanced cytotoxicity. Additionally, negatively charged blood components, mainly proteins, can compete with siRNA for binding to the cationic biopolymer and, thus, particles can disassemble into the bloodstream resulting in premature siRNA release followed by degradation and/or renal clearance. ${ }^{14}$ Thus, the chemical modification of chitosan appears as an interesting way to provide with new physicochemical characteristics to the chitosan biomolecule to overcome these pitfalls. Chitosan chains bear three reactive sites for chemical modification: two hydroxyl groups and one primary amine within the glucosamine units, which can be chemically modified to overcome barriers for gene delivery; however, for nucleic acid binding, the latter should be maintained. In this manner, several chitosan derivatives have emerged as potentially interesting alternatives for siRNA delivery, such as trimethyl-, ${ }^{15}$ thiolated-, ${ }^{16}$ glycol-, ${ }^{17}$ PEGylated-, ${ }^{18,19}$ guanidinylated-, ${ }^{20}$ or alkyl-chain-modified chitosans. ${ }^{21}$ All of these modifications resulted in improved particle solubility and stability, larger transfection efficiencies, and/or $\mathrm{pH}$ independence. Among these, hydrophobic modification of the biopolymer backbone appears particularly interesting since it has demonstrated great potential in enhancing the performance of gene nanovectors. For example, the transfection efficiency of siRNA into C2C12 cell lines using alkylated chitosans with different side-chain lengths was found to increase as the alkyl chain length increases up to ca. eight-carbon length. ${ }^{22}$ This higher transfection endowed by alkylated chitosans could be assigned, on the one hand, to the enhanced chitosan-siRNA complexation by the hydrophobicity-induced weakening of the electrostatic attractions between siRNA and the cationic nanocarrier and, on the other, to the larger nanovector uptake through the hydrophobic domains of cell membranes. ${ }^{22,23}$

This article describes the obtention of different alkyl-chainmodified chitosan nanovectors by the ionic gelation method and the role played by the aliphatic side-chain length and substitution degree in the loading, protection, stability, release profiles, transfection, and knockdown efficiencies of siRNA to construct suitable gene carriers. In particular, aliphatic side chains of different lengths (octyl, decyl, and dodecyl) were grafted to different extents in the polymer backbone to achieve several degrees of substitution. These modified chitosans were used to obtain chitosan-siRNA nanoparticles (CSNPs) by means of the ionic gelation process. It was observed that the incorporation of siRNA within the NPs was driven by a combination of electrostatic, hydrogen, and hydrophobic forces, as evidenced by isothermal titration experiments. This incorporation also led to additional changes in both particle sizes and surface charges. In this respect, unmodified CSNPs showed, in general, bulkier sizes than the corresponding nonderivatized unloaded counterparts (CNPs), whereas the opposite trend was found for the modified CSNPs. The hydrophobic modifications improved the siRNA loading and entrapment efficiency inside the particles, providing further protection from the surrounding medium, as well as an additional control over the cargo release kinetics by lowering the initial siRNA burst phase while keeping a sustained delivery for longer times. It was observed that hydrophobically modified CSNPs were fairly stable in physiologically relevant medium, with slight clustering upon long incubation. To test the cytotoxicity, transfection efficiency, and knockdown activity of the conceived NPs, a series of in vitro experiments were also performed in cervical cancer HeLa cells and RAW 264.7 macrophages, with an additional look at potential toxic and transfection efficiency differences depending on the cell type and physiology. ${ }^{24} \mathrm{HeLa}$ cells were more sensitive than macrophages to the presence of hydrophobically modified CSNPs in a dose-dependent manner. Impressively, CSNPs, comprising the hydrophobized chitosan, delivered siRNA inside the cell cytoplasm to a larger extent than their nonmodified counterparts thanks to their higher capability of cellular internalization. Afterward, these modified CSNPs manifested effective gene silencing, by inhibiting the expression 
of GFP overexpressed in modified HeLa cells, slightly greater than that of Lipofectamine 2000, a well-established transfection agent.

\section{EXPERIMENTAL SECTION}

2.1. Materials. Chitosan with an average molecular weight of $415000 \mathrm{~g} / \mathrm{mol}$ and $90 \%$ deacetylation degree (Fluka, middle-viscosity grade) was regularly used. Low- and highviscosity-grade chitosans, also from Fluka, were used for comparison. Sodium cyanohydroborate $\left(\mathrm{NaCNBH}_{4}\right)$; octyl, decyl, and dodecyl aldehydes; and pentasodium tripolyphosphate (TPP) were purchased from Sigma (Sigma-Aldrich Co.) and used as received. A BLOCK-iT control fluorescent oligo probe was obtained from Invitrogen (Carlsbad, MA). This fluorescein-labeled dsRNA oligomer has the same length, electrical charge, and structure as that of standard siRNA. UltraPure DNase/RNase-free distilled water was from Gibco (Life Technologies). All organic solvents were of highperformance liquid chromatography grade, and all other chemicals were of the highest purity commercially available.

2.2. Synthesis and Characterization of Chitosan Derivatives. Chitosan derivatives were obtained by a reductive amination process ${ }^{25-27}$ (see Scheme 1). As

Scheme 1. Scheme of the Alkylation Reaction Process for Derivatization of the Chitosan Backbone

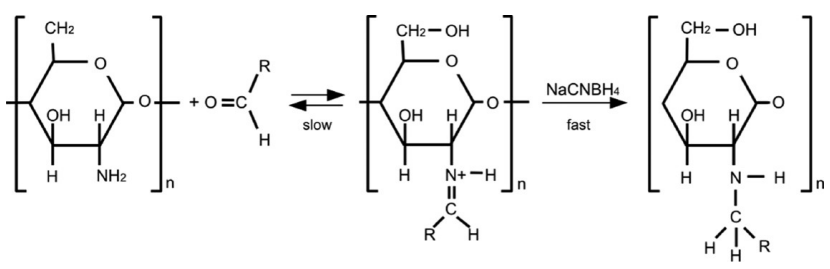

previously described, the alkylation reaction entailed grafting of alkyl side chains of 8,10 , and 12 carbons onto the chitosan backbone. ${ }^{25,28}$ Briefly, $2 \mathrm{~g}$ of chitosan was dissolved in $110 \mathrm{~mL}$ of $0.2 \mathrm{M}$ acetic acid. After dissolution, $75 \mathrm{~mL}$ of ethanol was added and $\mathrm{pH}$ was adjusted to 5.1 to avoid the precipitation of the biopolymer. Different aldehyde proportions $(5,10$, or $50 \%$ where corresponding) diluted in ethanol were added to the chitosan solution followed by a threefold molar excess of sodium cyanohydroborate. Next, the reaction was allowed to proceed under stirring for $24 \mathrm{~h}$ at room temperature. The alkylated chitosan was precipitated in ethanol, the $\mathrm{pH}$ adjusted to 7 and, then, the precipitate washed several times in ethanol/ water mixtures of increasing ethanol content from 70 to $100 \%$ $(\mathrm{v} / \mathrm{v})$.

Chitosan substitution degree was determined by ${ }^{1} \mathrm{H}$ nuclear magnetic resonance $\left({ }^{1} \mathrm{H}\right.$ NMR) spectroscopy (Figure $\mathrm{S} 1$ ) using a Bruker (XRD-500) instrument operating at $500 \mathrm{MHz}$ and $11.74 \mathrm{~T}$. The samples were prepared by dissolving $5 \mathrm{mg}$ of the different modified chitosans in $0.5 \mathrm{~mL}$ of a $2 \%(\mathrm{v} / \mathrm{v}) \mathrm{DCl} /$ $\mathrm{D}_{2} \mathrm{O}$ mixture at $\mathrm{pH}$ 3.2.

2.3. Preparation of Chitosan NPs. Chitosan nanoparticles (CNPs) and chitosan-siRNA nanoparticles (CSNPs) were prepared by ionic gelation through the crosslinking of TPP and chitosan chains, as previously described, ${ }^{29}$ with some modifications. Briefly, $1 \mathrm{mg} / \mathrm{mL}$ chitosan was dissolved in $0.2 \mathrm{M}$ acetic acid ( $\mathrm{pH} 4.2$ ) and stirred overnight at room temperature. Afterward, $0.5 \mathrm{mg}$ of TPP was dissolved in $0.75 \mathrm{~mL}$ of RNAse-free water and this mixture was directly dropped into the chitosan solution under constant stirring. For the production of CSNPs, a specific amount of siRNA was dissolved in $0.75 \mathrm{~mL}$ of TPP solution before its dropwise addition into chitosan one. Magnetic stirring was carried out at $700 \mathrm{rpm}$ at room temperature for $30 \mathrm{~min}$. The synthesized nanoparticles (NPs) were centrifuged at $15000 \mathrm{rpm}$ for 20 min and resuspended in RNAse-free distilled water. Finally, particle samples were filtered (Millipore Millex filters, $0.45 \mu \mathrm{m}$ pore size) and stored at $2-8{ }^{\circ} \mathrm{C}$.

2.4. Dynamic Light Scattering (DLS). Dynamic light scattering (DLS) measurements were performed using a static light scattering/DLS instrument (ALV-5000F, ALV-GmbH, Germany) equipped with a diode-pumped Nd:YAG solid-state laser (Coherent Inc., CA) supplying vertically polarized incident light $(\lambda=488 \mathrm{~nm})$ and an ALV SP-86 digital correlator. Measurements were made at a scattering angle of $\theta$ $=90^{\circ}$ to the incident beam with a duration of $2-5 \mathrm{~min}$. Prior to measurement, samples were filtered and thermally equilibrated for $10 \mathrm{~min}$. The intensity scale was calibrated against scattering from toluene, and each measurement was repeated at least three times. The obtained correlation functions were analyzed by the CONTIN method. ${ }^{30}$ Values of the apparent hydrodynamic radius $\left(R_{\mathrm{H}}\right)$ were calculated from the Stokes-Einstein equation

$$
D_{\mathrm{app}}=\frac{k_{\mathrm{B}} T}{6 \pi \eta R_{\mathrm{H}}}
$$

where $k_{\mathrm{B}}$ is the Boltzmann constant, $T$ the temperature, $\eta$ the solvent viscosity, and $D_{\text {app }}$ the apparent diffusion coefficient derived from the CONTIN analysis.

2.5. $\zeta$-Potentials. $\zeta$-Potentials of CNPs and CSNPs were measured using a Nano ZS-90 instrument (Nanoseries, Malvern Instruments, U.K.), which allows the analysis of particle electrophoretic mobilities. From this quantity, $\zeta$ potentials can be derived by means of the classical Smoluchowski equation with the Smoluchowski approximation (aqueous media and moderate electrolyte concentration)

$$
\alpha=\varepsilon \frac{\zeta}{\eta}
$$

where $\alpha, \varepsilon, \zeta$, and $\eta$ denote the electrophoretic mobility, permittivity of the media, $\zeta$-potential of the particles, and viscosity of the media, respectively. To perform the measurements, each particle solution was loaded into a capillary cell and thermally equilibrated at $25^{\circ} \mathrm{C}$. The number of runs was automatically set by the software $(>20)$, and the measurements were made at least in triplicate. Results were reported as the mean \pm standard deviation.

2.6. Transmission Electron Microscopy (TEM). To obtain TEM images, a drop of $5 \mu \mathrm{L}$ of CNP and CSNP suspensions was applied on the top of carbon-coated copper grids, blotted, washed, negatively stained with $2 \%(\mathrm{w} / \mathrm{v})$ phosphotungstic acid, air-dried, and examined with a JEOL JEM 1011 (Japan) transmission electron microscope at 120 $\mathrm{kV}$. Samples were diluted, when required, before deposition on the grids.

2.7. Quantitative Analysis of siRNA in CSNPs. To determine the encapsulation efficiency (EE) and loading capacity (LC) of the obtained nanocarriers, CSNPs were centrifuged at $15000 \mathrm{rpm}$ at $20{ }^{\circ} \mathrm{C}$ for $20 \mathrm{~min}$. Next, the siRNA concentration in the supernatant was determined by UV-vis and fluorescence spectroscopies using the supernatant 
of each CNP as a blank. UV-vis plots were made at $260 \mathrm{~nm}$ subtracting the potential contribution at $280 \mathrm{~nm}$ from small protein residues in the solution media. Fluorescence spectra were recorded by setting $\lambda_{\mathrm{ex}}=494 \mathrm{~nm}$ and $\lambda_{\mathrm{em}}=519 \mathrm{~nm}$. UVvis spectra were recorded using a Cary Bio 100 UV-vis spectrophotometer (Agilent Technologies), whereas fluorescence spectra were recorded using a Cary Eclipse spectrophotometer (Agilent Technologies). Each sample was measured in triplicate from three different particle batches, and the final results were averaged. The EE and LC were calculated by the following expressions

$$
\begin{aligned}
\mathrm{EE}(\%) & \\
= & \frac{\text { totalamountofSiRNAfeeded }- \text { siRNAinsupernatant }}{\text { totalamountofSiRNAfeeded }} \\
& \times 100 \quad \text { totalweightofnanoparticles } \\
= & \frac{\text { totalamountofSiRNAfeeded }- \text { siRNAinsupernatant }}{\text { to }} \\
& \times 100
\end{aligned}
$$

2.8. Binding Efficiency Assay. Agarose gel electrophoresis was used to determine the successful binding of siRNA to chitosan. The different modified chitosan-based nanovectors encapsulating an initial feed of $150 \mathrm{pmol}$ of siRNA were loaded in each well $(20 \mu \mathrm{L})$. Gel electrophoresis experiments were performed at a constant voltage of $150 \mathrm{~V}$ during 30 min using 2\% agarose gels. siRNA was stained with SYBR Green I (Life Technologies, Thermo Fisher Scientific) in a 1:10 000 dilution in TE Buffer ( $10 \mathrm{mM}$ Tris $\cdot \mathrm{HCl}, \mathrm{pH}, 1$ $\mathrm{mM}$ sodium ethylenediaminetetraacetate (EDTA)) for $15 \mathrm{~min}$. An E-Gel Safe Imager Real-Time Transilluminator and an EGel iBase Power System (Invitrogen, Thermo Fisher Scientific) were used to visualize the samples. Photographs were taken with a CANON IXUS 155 camera.

2.9. siRNA Serum Stability Assay. Following a previously reported protocol, ${ }^{10}$ CSNPs loaded with 150 pmol of siRNA were incubated at $37{ }^{\circ} \mathrm{C}$ in Dulbecco's modified Eagle's medium (DMEM) supplemented with 5 and $50 \%(\mathrm{v} / \mathrm{v})$ of fetal bovine serum (FBS). At different times (0, 0.5, 2, 4, 7, 24, 48 , and $72 \mathrm{~h}$ ), $100 \mu \mathrm{L}$ of each particle's solution was removed and stored at $-20{ }^{\circ} \mathrm{C}$ until gel electrophoresis was performed. To carry out the experiments, the serum activity was first stopped. To do that, samples were incubated at $80{ }^{\circ} \mathrm{C}$ for 10 min and, then, $5 \mu \mathrm{L}$ of heparin $(1000 \mathrm{U} / \mathrm{mL})$ was added for displacing the siRNA from CSNPs. The analysis of siRNA integrity was carried out using a $4 \%$ agarose gel, as described above.

2.10. Isothermal Titration Calorimetry (ITC). The interactions and binding between siRNA and the different modified chitosans were analyzed using a VP-ITC titration microcalorimeter (MicroCal Inc., Northampton, MA) at 25 ${ }^{\circ} \mathrm{C}$. The sample and reference cells of the calorimeter were filled with chitosan (1.06 $\mu \mathrm{M}, 10 \mathrm{mM}$ acetate buffer, $\mathrm{pH} 4.8)$ and pure buffer solutions, respectively. Next, the anionic solution containing siRNA $(2 \mu \mathrm{M})$ and TPP $(2.88 \mathrm{mM})$ dissolved in RNAse-free water was introduced into the thermostated sample cell using a computer-controlled syringe and stirred at $350 \mathrm{rpm}$. Each experiment consisted of an initial $2 \mu \mathrm{L}$ injection (neglected in the analysis) followed by other 27 $10 \mu \mathrm{L}$ injections with a spacing time of $600 \mathrm{~s}$, which is enough time for the heat signal to return to the baseline. The ITC plots represent the heats of injection normalized by the siRNA concentration added per injection, $Q^{*}$, as a function of the siRNA/chitosan molar ratio. The heats of dilution corresponding to the titration of the anionic solution into pure acetate buffer were subtracted to obtain the net binding heats. All experiments were carried out in triplicate.

The raw ITC data were analyzed as described previously. ${ }^{31,32}$ Briefly, the binding isotherms were fitted to a twobinding-site model, which makes use of the Langmuir isotherm binding equilibrium for two independent types of associations

$$
Q=M \cdot V \cdot\left(n_{1} \cdot \theta_{1} \cdot \Delta H_{1}+n_{2} \cdot \theta_{2} \cdot \Delta H_{2}\right)
$$

where $Q$ is the heat per injection; $M$ the macromolecule concentration (in this case, siRNA); $V$ the cell volume; $n$ and $\Delta H$ are the stoichiometry and enthalpy of interactions, respectively; $\theta$ the fraction of ligand bound to the macromolecule; and the subindices 1 and 2 stand for the two sets of sites.

Using a nonlinear fitting algorithm, the former equation can be solved for $\theta_{1}$ and $\theta_{2}$ using the equilibrium equations for binding constants $K_{1}$ and $K_{2}$ as

$$
\begin{aligned}
K_{1} & =\frac{\theta_{1}}{\left(1-\theta_{1}\right)[\mathrm{X}]}, K_{2} \\
& =\frac{\theta_{2}}{\left(1-\theta_{2}\right)[\mathrm{X}]}[\mathrm{X}]=X-M\left(n_{1} \cdot \theta_{1}+n_{2} \cdot \theta_{2}\right)
\end{aligned}
$$

where $X$ is the total ligand concentration and $[\mathrm{X}]$ the concentration of the free ligand (chitosan).

2.11. Colloidal Stability. The colloidal stabilities of CNPs and CSNPs were assessed by diluting the particles $(1 / 50)$ at 37 ${ }^{\circ} \mathrm{C}$ under different solution conditions (aqueous solutions of different $\mathrm{pH}$ values ranging from 3 to 9; Dulbecco's modified Eagle's medium (DMEM) supplemented with $10 \% \mathrm{FBS}$; and RNAse-free water), ${ }^{10}$ and moderate stirring for 5 days. $\zeta$ Potential and hydrodynamic sizes were acquired using a Zetasizer Nano ZS-90 (Malvern Instruments, U.K.). Experiments were performed in triplicate.

2.12. In Vitro Release Studies. siRNA release from CSNPs was measured in vitro at a constant temperature of 37 ${ }^{\circ} \mathrm{C}$ under magnetic stirring (300 rpm) for several days at three different $\mathrm{pH}$ values $(7.4,5.0$ simulating the acidic microenvironment of the cytoplasm, ${ }^{33}$ and $\mathrm{pH} 2.0$, lying in the range of gastric $\mathrm{pH}){ }^{34}$ The released siRNA concentration was determined at different time intervals for every solution $\mathrm{pH}$ by placing $1 \mathrm{~mL}$ of CSNPs into dialysis tubes (Spectra/Por, MWCO 3500, SpectraLabs) immersed in $50 \mathrm{~mL}$ of buffer supplemented with $10 \%$ fetal bovine serum (FBS) at the $\mathrm{pH}$ of interest. At each sampling time, $1 \mathrm{~mL}$ of the medium was withdrawn and replaced with the same volume of fresh buffer to maintain the required sink conditions. The siRNA content in the supernatant was measured by means of UV-vis and fluorescence spectroscopies using calibration curves in the corresponding buffers, as previously described. Assays were carried out in triplicate.

2.13. Cell Culture. HeLa cervical cancer cells and HeLaGFP-modified cells (Cell Biolabs, San Diego, CA) were grown at standard culture conditions $\left(5 \% \mathrm{CO}_{2}\right.$ at $\left.37{ }^{\circ} \mathrm{C}\right)$ in DMEM supplemented with $10 \%$ (v/v) FBS, $2 \mathrm{mM}$ L-glutamine, $1 \%$ (v/ v) penicillin/streptomycin, $1 \mathrm{mM}$ sodium pyruvate, and 0.1 mM MEM nonessential amino acids (NEAAs). RAW 264.7 macrophages were cultured in 50:50 DMEM/F12-Ham mixed 
medium supplemented with $10 \%(\mathrm{v} / \mathrm{v})$ FBS, $2 \mathrm{mM} \mathrm{L-}$ glutamine, $1 \%(\mathrm{v} / \mathrm{v})$ penicillin/streptomycin, $1 \mathrm{mM}$ sodium pyruvate, and $0.1 \mathrm{mM} \mathrm{NEAA}\left(37^{\circ} \mathrm{C}, 5 \% \mathrm{CO}_{2}\right.$, in humidified atmosphere).

2.14. In Vitro Cell Cytotoxicity. The cytotoxicity of CSNPs was assayed in vitro using the cell counting kit-8 (CCK-8) cytotoxicity assay. Cervical HeLa cancer cells and RAW 264.7 macrophages at a confluence of $80-90 \%$ were seeded into 96-well plates $\left(100 \mu \mathrm{L}, 1.5 \times 10^{4}\right.$ cells/well $)$ and grown for $24 \mathrm{~h}$ at standard culture conditions. After $24 \mathrm{~h}, 100$ $\mu \mathrm{L}$ of known CNPs and CSNPs concentrations diluted in the corresponding cell culture media was injected into the wells and incubated for 24 and $48 \mathrm{~h}$, respectively. Some cells were transfected with $1 \mathrm{mM}$ siRNA BLOCK-iT control fluorescent oligo (Invitrogen) using Lipofectamine 2000 as a positive control (Life Technologies, Carlsbad, CA) following the manufacturer's instructions. Some wells were also left without NPs as a negative control (blank). After the corresponding incubation time, the culture medium was discarded, cells washed with $10 \mathrm{mM}$ phosphate-buffered saline (PBS) ( $\mathrm{pH}$ 7.4) several times, and new fresh medium $(100 \mu \mathrm{L})$ added. Next, $10 \mu \mathrm{L}$ of CCK-8 reagent was injected into each well. After $2 \mathrm{~h}$, the absorption at $450 \mathrm{~nm}$ was measured with an UV-vis microplate absorbance reader (Bio-Rad model 689). Cell viability, that is, survival rate (SR), was calculated as follows

$$
\mathrm{SR}(\%)=\frac{\mathrm{Abs}_{\text {sample }}}{\mathrm{Abs}_{\text {blank }}} \times 100
$$

where $\mathrm{Abs}_{\text {sample }}$ is the absorbance at $450 \mathrm{~nm}$ for full samples and $\mathrm{Abs}_{\text {blank }}$ is the absorbance in the absence of NPs.

2.15. Cellular Uptake and Gene Silencing by Fluorescence Microscopy. CSNP cell internalization was monitored by confocal microscopy by seeding HeLa, HeLaGFP, and RAW 264.7 cells on poly-L-lysine-coated glass coverslips $\left(12 \times 12 \mathrm{~mm}^{2}\right)$ placed inside six-well plates $(3 \mathrm{~mL}, 5$ $\times 10^{4}$ cells per well grown for $24 \mathrm{~h}$ at standard culture conditions). Then, $250 \mu \mathrm{L}$ corresponding to $0.5 \mathrm{mg}$ of NPs containing ca. 37 pmol of siRNA was added. After incubation, the NP-containing cells were washed three times with PBS ( $\mathrm{pH} 7.4)$ and, then, fixed with paraformaldehyde $4 \%(\mathrm{w} / \mathrm{v})$ for $10 \mathrm{~min}$, washed with PBS, permeabilized with $0.2 \%(\mathrm{w} / \mathrm{v})$ Triton X-100, and stained with 4,4-difluoro-4-bora-3a,4a-diazas-indacene (BODIPY) Phalloidin (Invitrogen). Afterward, cells were washed again with PBS, mounted on glass slides stained with ProLong Gold antifade 4',6-diamidino-2-phenylindole (DAPI) (Invitrogen), and kept for $24 \mathrm{~h}$ at $-20{ }^{\circ} \mathrm{C}$. Samples were visualized with a $63 \times$ objective using an epifluorescence microscope Leica DMI6000B equipped with a Leica AF6000 modular system and a DFC3665FX camera (Leica Microsystems $\mathrm{GmbH}$, Heidelberg, Mannheim, Germany). In the obtained images, the blue channel corresponded to DAPI $\left(\lambda_{\mathrm{ex}}\right.$ $=355 \mathrm{~nm})$, the red one to BODIPY Phalloidin $\left(\lambda_{\mathrm{ex}}=633 \mathrm{~nm}\right)$, and the transmitted light was obtained in differential interference contrast mode. Finally, the green channel corresponded to the excitation of the fluorescein isothiocyanate (FITC)-doped siRNA fluorescent oligo (Invitrogen). The same channel was used for the excitation at $355 \mathrm{~nm}$ of the green fluorescent protein in HeLa-GFP cells.

2.16. Cellular Uptake and Gene Silencing by Flow Cytometry. The cellular siRNA uptake in HeLa cells was additionally quantified by measuring the fluorescence of a
FITC-labeled ds-siRNA oligo (Invitrogen) encapsulated into CSNPs by flow cytometry. HeLa cells expressing GFP were used to investigate the gene-silencing efficiency of CSNPs loaded with a specific siRNA inhibiting the fluorescence of overexpressed GFP (from Ambion). Normal HeLa cells were used as a negative control, and nontransfected HeLa-GFP cells were used as a positive one. siRNA transfected with Lipofectamine 2000 was used as an additional transfection reference. Cells $\left(2 \times 10^{5}\right.$ per well $)$ were washed once with PBS and, then, harvested with $0.05 \%$ trypsin $/ 0.025 \%$ EDTA followed by several washes at $1200 \mathrm{rpm}$ for $4 \mathrm{~min}$ and resuspension in $1 \mathrm{~mL}$ of PBS. The gene-silencing efficiency was determined by collecting fluorescence data through flow cytometry (BD FACSAria IIu, BD, Franklin Lakes, NJ). In all analyses, $2 \times 10^{5}$ events were acquired, scored using a FACSAria II analyzer (BD, Franklin Lakes, NJ), and processed by the PC FACSDiva software program (BD, Franklin Lakes, NJ). Cell count was always performed per $10^{6}$ events. Transfection efficiency was expressed as the percentage of the fluorescence intensity of gathered cells compared to the total fluorescence intensity of nontransfected HeLa-GFP cells (positive control).

\section{RESULTS AND DISCUSSION}

3.1. Preparation and Characterization of Chitosan NPs. Chitosan NPs were prepared by means of the ionotropic gelation method. This procedure does not use organic solvents or sonication (although optional) common to other preparative methods, which may be harmful for peptides and proteins by altering their structural conformations (see Supporting Information for details). For NP formation, the $\mathrm{p} K_{\mathrm{a}}$ of amino groups of the derivatized chitosans used herein ranged from 6.5 to 6.8. Therefore, more than $90 \%$ of amines were protonated and positively charged at $\mathrm{pH}$ 5.5. The $\mathrm{p} K_{\mathrm{a}}$ values of phosphate groups of siRNA were close to zero, ${ }^{35}$ so more than $99 \%$ were negatively charged at $\mathrm{pH}$ above 2.0 . The physicochemical characterization of chitosan (CNPs) and chitosan-siRNA (CSNPs) NPs was investigated using an unmodified medium-molecular-weight chitosan (CM), and the same biopolymer was derivatized with octyl aldehyde or dodecyl aldehyde chains with a 5, 10, and 50\% degree of substitution on the polymer backbone, unless otherwise stated. This choice was based on the sizes and $\zeta$-potentials of the obtained NPs (see Figure S2), which should ensure a good colloidal stability, long circulation times, and enhanced passive accumulation at the target site. Moreover, CNPs and CSNPs, based on a chitosan derivatized with decyl aldehyde at a substitution degree of $10 \%$, were also prepared to compare the effect of the hydrophobic chain length with a determined grafting density on the physicochemical properties of the complex particles formed. The nomenclature used to label the different chitosan-based NPs is summarized in Table S1.

To determine the influence of the substitution of hydrophobic chains on the size and surface electrical charge of CNPs, DLS and $\zeta$-potential data were obtained at $37{ }^{\circ} \mathrm{C}$. Furthermore, TEM images were acquired to get insight into the NP morphology. Some differences in $\zeta$-potentials and particle sizes arose when the chitosan backbone was modified to different extents with aliphatic chains of several lengths, as observed in Figure 1. For CNPs containing derivatized chitosan with a $5 \%$ substitution degree, $\zeta$-potentials slightly increased as the aliphatic chain length increased. Conversely, the opposite trend was observed at higher substitution degrees. 

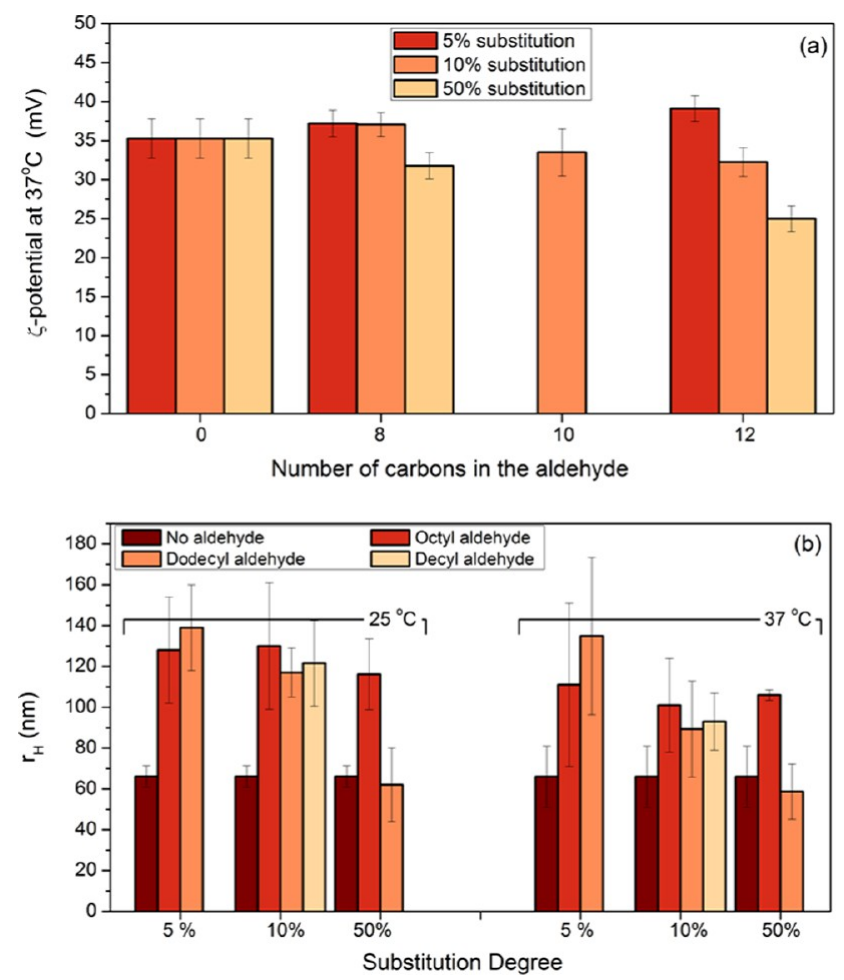

Figure 1. (a) $\zeta$-Potentials of derivatized CNPs at $37^{\circ} \mathrm{C}$ for 0,810 , and 12 carbons in the aldehyde side chains with 5,10 , and $50 \%$ of substitution degrees. (b) Hydrodynamic radii of unmodified, 8carbon, 10-carbon, and 12-carbon derivatized CNPs at 25 and $37^{\circ} \mathrm{C}$.

This can be explained based on the assumption that at lower substitution degrees, the uncharged, hydrophobized side chains of chitosan might have preferably arranged themselves toward the interior of CNPs, allowing a relatively larger exposure of their positively charged amine groups to the particles' surface. In other words, when the substitution degree increased, lower surface charges of CNPs were noted as a consequence of (i) the reduction in the number of protonated amines upon substitution, ${ }^{26,27}$ and/or (ii) the potential presence of side chains at the NP surfaces due to packing constraints, which additionally screened the cationic surface charges.

The average sizes of nonhydrophobized CNPs $\left(r_{\mathrm{h}}=66 \pm 5\right.$ $\mathrm{nm}$ ) were smaller than those of the hydrophobized ones (see Figure $1 \mathrm{~b}$ ), which ranged from ca. 70 to $140 \mathrm{~nm}$ depending on the side-chain length and substitution degree. ${ }^{36}$ The hydrophobic segments of derivatized chitosan of CNPs might have pointed inward, giving rise to denser cores and increase in size. This effect was more evident for CNPs made of derivatized chitosan with the lowest degree of substitution (5\%), provided that all aliphatic chains were anchored in the NP cores, in agreement with $\zeta$-potential values. In contrast, particle sizes generally decreased as the degree of substitution increased, seemingly as a consequence of a stronger compaction of CNPs cores to avoid the exposure of their hydrophobic side chains to water, especially noticeable for those bearing 12-carbon ones. Since the complete packing of substituting side chains inside the particle cores is not probable, a rearrangement of some of these on/near the NP surfaces might have also taken place, which is in agreement with $\zeta$-potential data. On the other hand, it was observed that the aliphatic chain length played a key role in determining NP size, particularly for those particles containing derivatized chitosan with high degrees of substitution, which had smaller sizes. ${ }^{25,28}$ Moreover, particle hydrodynamic radii were observed to decrease by ca. $30 \mathrm{~nm}$ when the solution temperature increased from 25 to $37^{\circ} \mathrm{C}$. It is worth mentioning that previous works have shown larger chitosan-based NPs produced by means of ionotropic gelation than those obtained in this work. ${ }^{22,36,37}$

TEM images of CNPs (Figure 2) were obtained for the different types of derivatized chitosans. The CNP morphology was almost spherical, especially for unmodified CNPs, with some surface roughness. This effect was more clearly seen for CNPs bearing substituted short-length side chains, which displayed a certain hairy appearance (Figure $2 \mathrm{~d}-\mathrm{f}$ ) in contrast to those bearing 12-carbon ones, displaying smoother surfaces. CNPs' average sizes were in agreement with those obtained by DLS.

3.2. Energetics of CSNP Formation. The capability of any given delivery system to avoid premature dissociation and promote cargo release to the target organ or tissue is strongly related to the binding affinity between the therapeutic macromolecule and the selected vector. Moreover, the addition of siRNA together with TPP molecules during the ionic gelation process has previously led to better gene-silencing activities compared to other methods for particle/polyplex formation. ${ }^{10}$ An effective way to assess the involved interactions is to measure the heats produced/absorbed in such a complexation process and evaluate the associated binding thermodynamic quantities. To this purpose, ITC has become one of the most useful techniques to analyze the type and extent of interactions playing a role upon complexation of siRNA and subsequent NP formation. To determine the siRNA/chitosan heats of binding in the presence of the TPP cross-linker during the NP formation process, two sets of experiments were carried out; that is, anionic solutions containing siRNA with TPP and only TPP (as a control) were titrated into the sample cell containing a chitosan solution. Then, the net heats of interaction between siRNA and chitosan along the NP formation process were obtained after subtraction of the heats evolved from the control experiment. In this way, heats from siRNA dilution and TPP-chitosan interactions were somehow neglected. The influence of the nature and degree of substitution in the chitosan backbone on the energetics of NP formation and siRNA entrapment was also assessed by selecting the following chitosan types as components of the NPs: $\mathrm{CM}, \mathrm{C} 8 \mathrm{Mb}, \mathrm{C} 8 \mathrm{Mc}$, and $\mathrm{C} 12 \mathrm{Mc}$.

Figure 3 shows the net heats of interaction upon injection of a siRNA + TPP solution into chitosan solutions (after subtraction of the heat evolved after the titration of TPP into the same chitosan solution) as a function of the siRNA/ chitosan molar ratio for different types of hydrophobically modified chitosans. The solid lines represent the fittings to the experimental data. Table 1 shows the derived thermodynamic parameters. From Figure 3, siRNA/chitosan interactions are characterized by initial electrostatic interactions and hydrogen bonding between the biopolymer and siRNA as shown by the negative values of the heats of interactions $\left(\Delta H_{\mathrm{i}}\right.$, where the subscript denotes the binding site; see below). $\Delta H_{\mathrm{i}}$ progressively increased, becoming less negative, as the siRNA/chitosan molar ratio increased. This finding is consistent with those reported for other systems such as protein-drug ${ }^{28,31,38}$ and polyelectrolyte-polyelectrolyte systems, such as chitosan-DNA interactions. ${ }^{32}$ As the siRNA/ chitosan molar ratio further increased, the evolved heats 

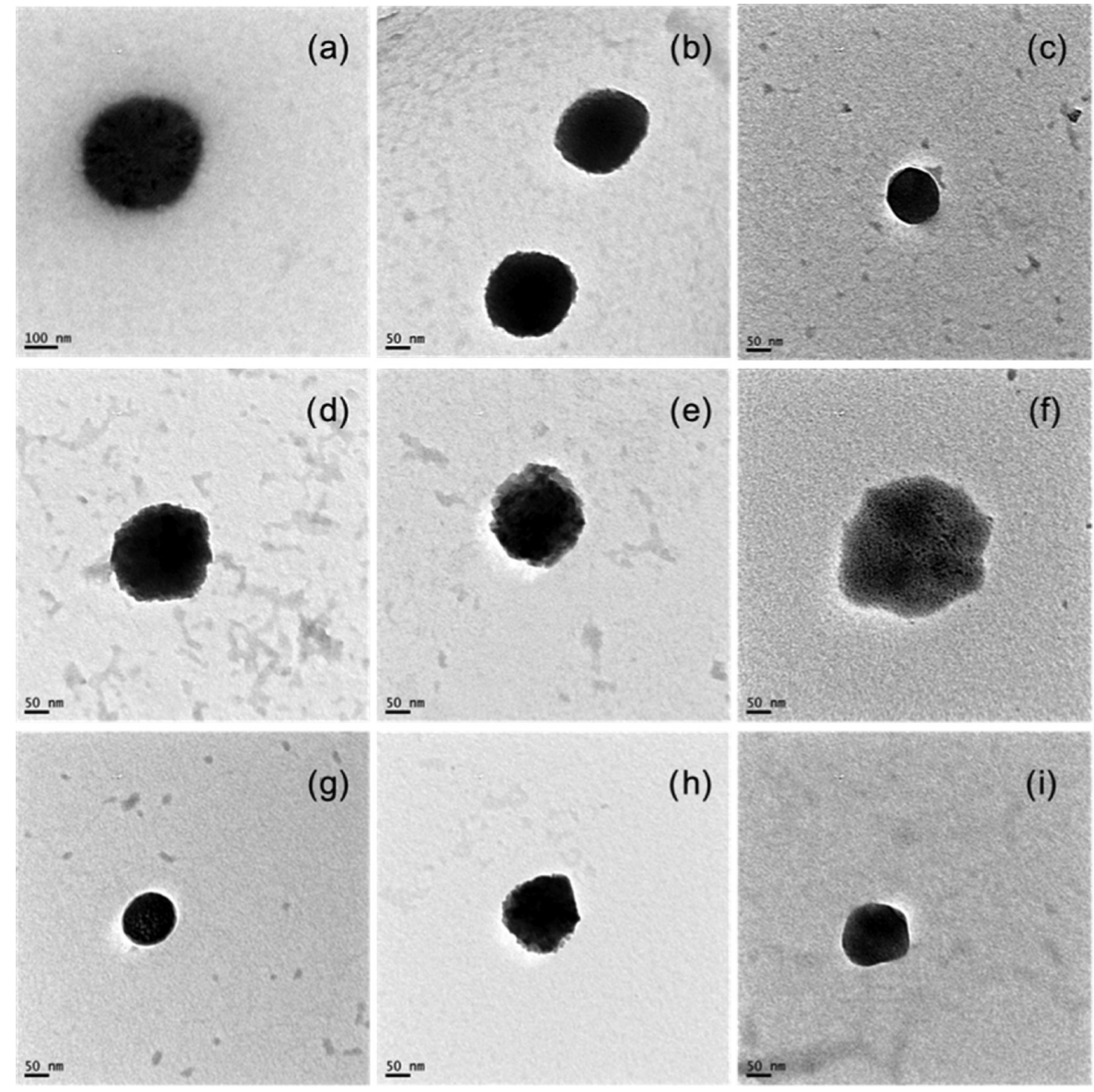

Figure 2. TEM images of CNPs obtained from (a) CL, (b) CM, (c) CH, (d) C8Ma, (e) C8Mb, (f) C8Mc, (g) C12Ma, (h) C12Mb, and (i) $\mathrm{C} 12 \mathrm{Mc}$ chitosans. Sample nomenclature corresponds to that stated in Table 1.

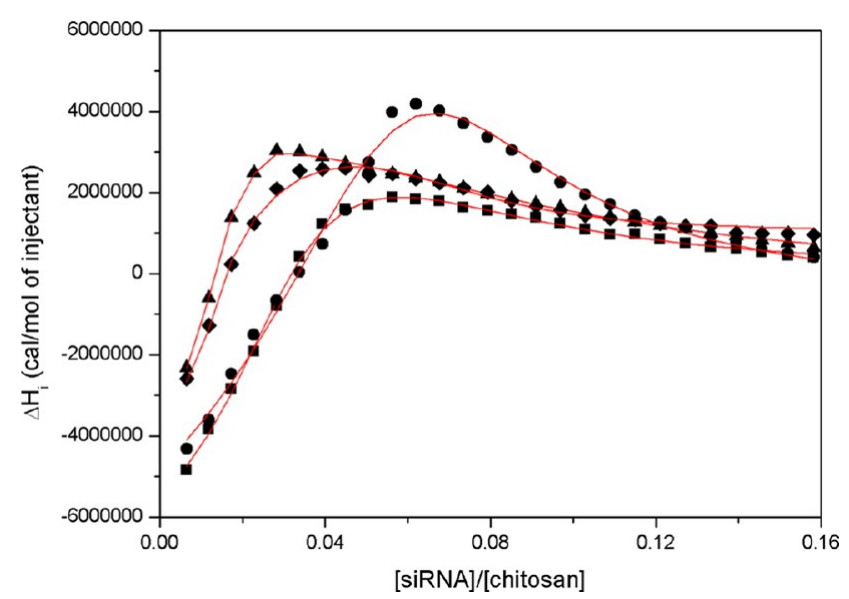

Figure 3. ITC experiments showing the evolution of the heats of interaction as a function of the [siRNA]/[chitosan] molar ratio for (অ) $\mathrm{CM},(\bullet) \mathrm{C} 8 \mathrm{Mb},(\boldsymbol{\Delta}) \mathrm{C} 8 \mathrm{Mc}$, and $(\bullet) \mathrm{C} 12 \mathrm{Mc}$ at $25{ }^{\circ} \mathrm{C}$ in aqueous solution. Red lines represent the fitting of experimental data to the two-biding-site model used.

became positive (ca. 0.02-0.04), depending on the type of chitosan, and then passed through an endothermic maximum to subsequently decrease again. In this regard, the increasing positive $\Delta Q_{i}$ values may be ascribed to the dehydration of chitosan and siRNA chains, the charge-shielding effects upon mutual interaction, and the important role of hydrophobic interactions. Furthermore, the subsequent endothermic decrease might be generated by dipole-dipole interactions of water molecules favorably oriented toward adjacent formed NPs, as observed for other systems. ${ }^{39}$ Finally, a plateau region was observed at the largest siRNA/chitosan molar ratios (>0.14) corresponding to dilution effects.

Regarding the thermodynamic characterization, Table 1 summarizes the enthalpy, entropy, binding constant, and stoichiometry of siRNA/chitosan binding derived on the basis of the two-binding-sites model. As observed, binding of siRNA to the differently modified chitosans involved binding constants on the order of ca. $10^{7}-10^{8}$ and $10^{6}-10^{7}$ for the first and second classes of binding sites, respectively. It was noticed that the shorter the side chain of the biopolymeric backbone, the larger the binding constant was. This could be attributed to the higher availability of free amine groups to electrostatically interact with the phosphate ones of siRNA in chitosan derivatives bearing shorter side chains and lower extents of substitution. It is worth mentioning that much lower levels of second-class binding constants might be expected, as a consequence of superimposed steric restrictions. However, the large enhancement of hydrophobic interactions partially compensates this point.

The stoichiometry of binding is fractional ( $n$ values less than 1.0) corresponding to the interaction of multivalent substrate/ ligands. ${ }^{40}$ Concerning the enthalpy of interaction, the relatively high exothermic enthalpies for the first class of binding sites denote the predominance of hydrogen and electrostatic interactions, whereas the large positive enthalpies for the 
Table 1. Thermodynamic Parameters of Interaction between siRNA and Chitosan upon the Formation of CSNPs ${ }^{a}$

$\begin{array}{lcc}\mathrm{CSNP} & 10^{8} K_{\mathrm{i}}\left(\mathrm{M}^{-1}\right) & 10^{-2} n_{\mathrm{i}} \\ \mathrm{CM} & 2.8 \pm 0.5 & 2.6 \pm 0.3 \\ & 0.3 \pm 0.01 & 3.4 \pm 0.7 \\ \mathrm{C} 8 \mathrm{Mb} & 1.4 \pm 0.2 & 7.7 \pm 0.5 \\ & 0.5 \pm 0.1 & 0.06 \pm 0.01 \\ \mathrm{C} 8 \mathrm{Mc} & 1.3 \pm 0.1 & 0.2 \pm 0.3 \\ & 0.2 \pm 0.1 & 5.1 \pm 0.8 \\ \mathrm{C} 12 \mathrm{Mc} & 0.6 \pm 0.1 & 0.6 \pm 0.2 \\ & 0.07 \pm 0.01 & 1.9 \pm 0.06\end{array}$

$\begin{aligned} & 10^{3} \Delta H_{\mathrm{i}}(\mathrm{kcal} / \mathrm{mol}) \\ &-7.4 \pm 0.4 \\ & 2.2 \pm 0.3 \\ &-6.8 \pm 0.5 \\ & 4.2 \pm 0.2 \\ &-4.3 \pm 0.2 \\ & 3.1 \pm 0.1 \\ &-4.9 \pm 0.3 \\ & 2.8 \pm 0.4\end{aligned}$
$\Delta S_{\mathrm{i}}(\mathrm{kcal} /(\mathrm{mol} \mathrm{K}))$
$-24.8 \pm 3.2$
$7.5 \pm 0.6$
$-22.5 \pm 2.1$
$14.2 \pm 0.3$
$-14.2 \pm 1.6$
$10.5 \pm 1.7$
$-16.3 \pm 2.3$
$9.4 \pm 1.4$

${ }^{a} K$, binding equilibrium constant; $n$, binding stoichiometry; $\Delta H$, enthalpy changes; $\Delta S$, entropy changes.
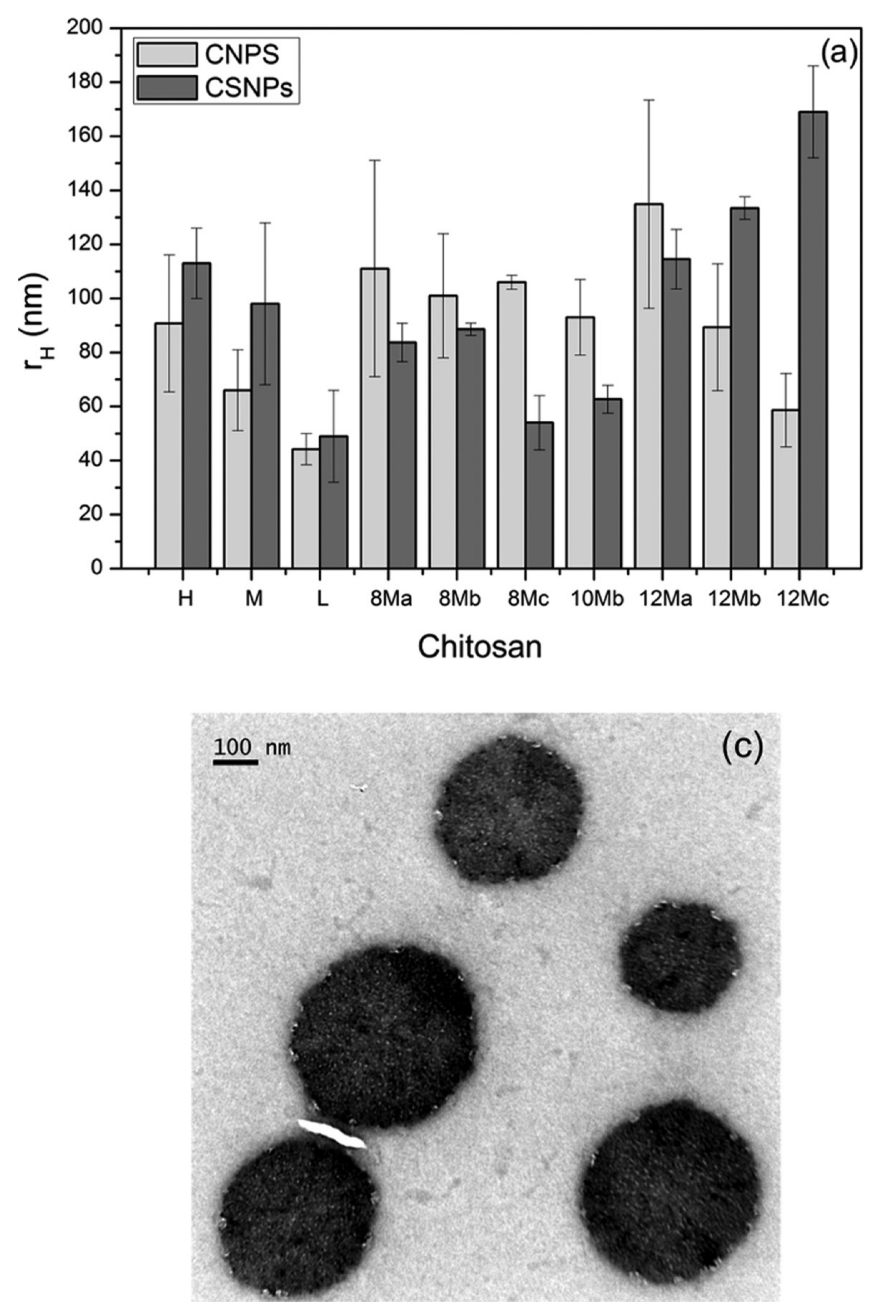
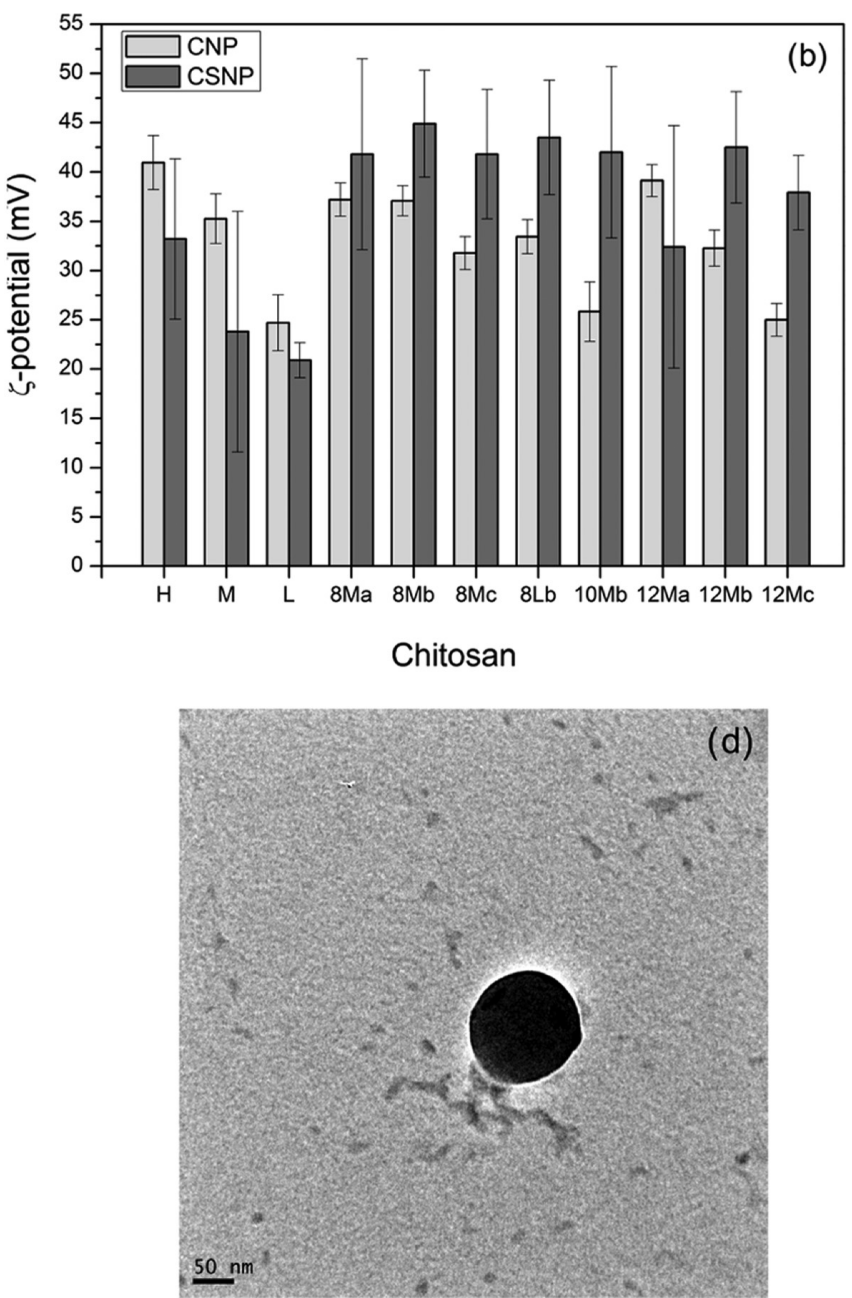

Figure 4. (a) Hydrodynamic radii and (b) $\zeta$-potentials of CNPs and CSNPs in aqueous solution. TEM images of CSNPs loaded with 150 pmol of siRNA for (c) $12 \mathrm{Mc}$ and (d) $8 \mathrm{Mb}$.

second class point to the large predominance of hydrophobic forces in the binding process. This was additionally verified by the change in the entropy of binding, which pointed to a clear enthalpy-driven process for the early stages of the binding process (shown by negative values in $\Delta S_{\mathrm{i}}$ ) and an entropically driven one for the second set of binding sites as shown by the positive values observed. This behavior might be related to the release of water molecules to the solvent and molecular rearrangements of the polymeric chains within the modified CNPs at this stage.

3.3. siRNA Entrapment inside Chitosan NPs. To elucidate the optimal siRNA loading extent inside the biopolymeric particles for effective silencing, we analyzed the entrapment efficiencies (EEs) and loading capacities (LCs) in the derivatized chitosan-based NPs at different initial siRNA concentrations. Four initial amounts $(50,100,150$, and 250 pmol) of the genetic material with the different hydrophobized chitosans were mixed with the TPP solution to obtain CSNPs following the methodology described in the Section 2. From Table S2 (see SI), it can be observed that the lower the siRNA amount fed, the lower the LC obtained as a result of the uncompleted saturation of NPs within the concentration range analyzed. In addition, lower EE and LC values were obtained for nonderivatized CSNPs compared to those for the hydrophobized ones; those formed by medium-molecularweight chitosan were the most effective, especially at the lowest 
Scheme 2. Scheme Depicting Possible siRNA and Side-Chain Distribution within CSNPs

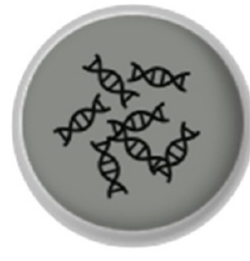

Unmodified Chitosan

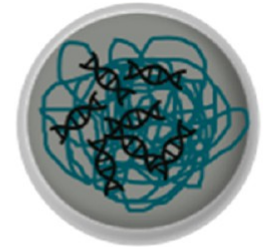

8-carbons length modified Chitosan

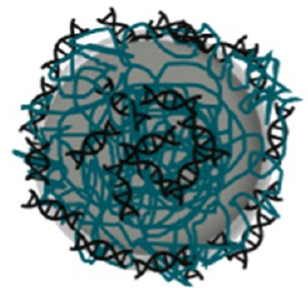

12-carbons length modified Chitosan
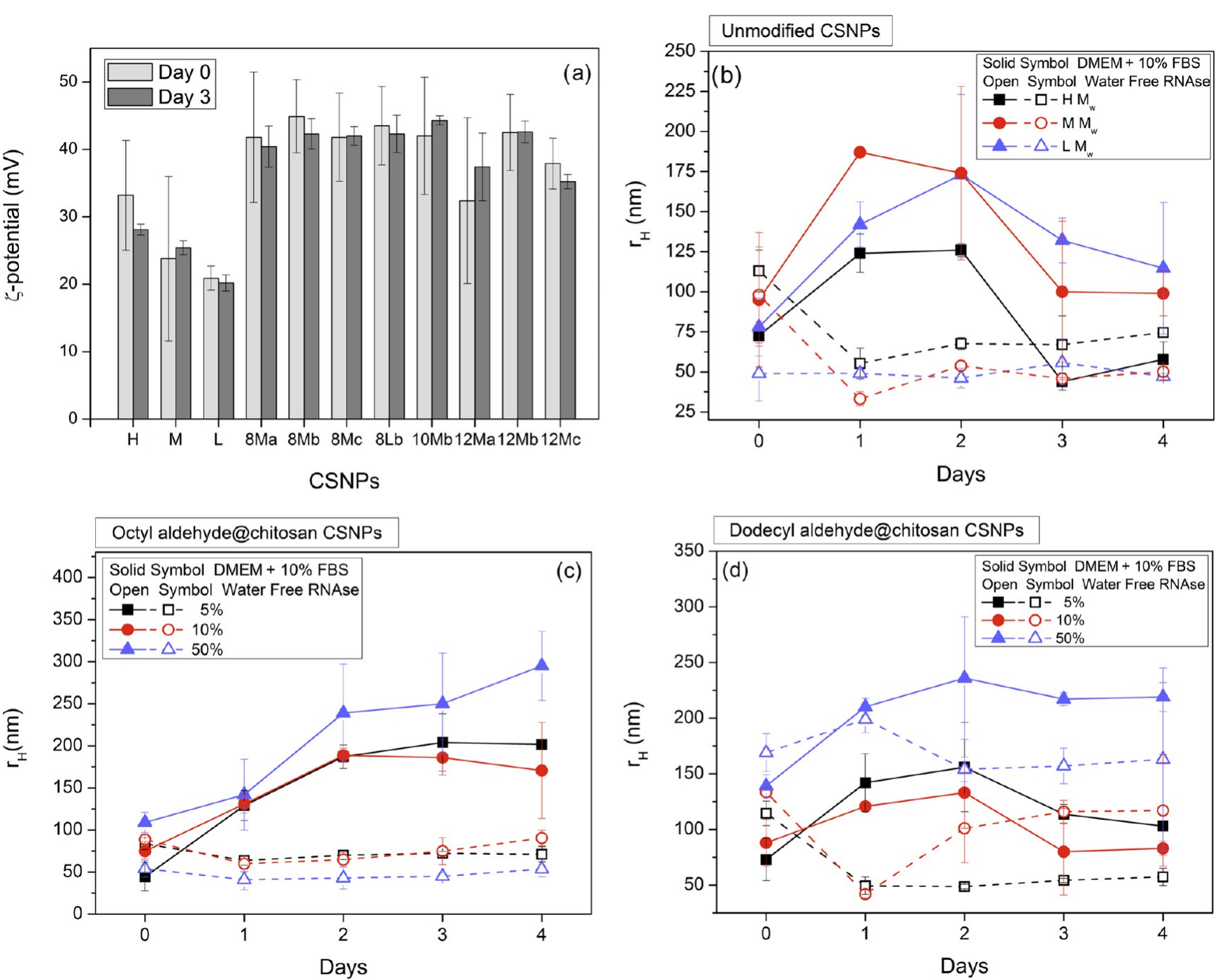

Figure 5. (a) Temporal evolution of $\zeta$-potentials of CSNPs for 3 days in RNAse-free water at $37^{\circ} \mathrm{C}$. (b-d) Temporal size evolution of CSNPs for 5 days at $37^{\circ} \mathrm{C}$ in RNAse-free water (open symbols) and DMEM supplemented with 10\% FBS (solid symbols). (b) NPs containing unmodified chitosans with $(\mathbf{\square}, \square)$ high, $(\boldsymbol{\bullet}, \mathrm{O})$ medium, and $(\boldsymbol{\Delta}, \triangle)$ low $M_{\mathrm{w} . .}$ (c) NPs containing 8-carbon and (d) 12-carbon derivatized chitosans with ( $\square) 5 \%,(\bullet, \bigcirc) 10 \%$, and $(\boldsymbol{\Delta}, \triangle) 50 \%$ degree of substitution.

siRNA amounts fed. Although a decrease in LC and EE might be expected when the number of free amino groups is reduced upon aliphatic side-chain substitution, these quantities strikingly increased upon chitosan derivatization. For example, it is remarkable that EEs for hydrophobized CSNPs were twofold higher than those of nonmodified ones. Hence, hydrophobic modifications could improve the gene loading/ entrapment capacity and might provide further control over the cargo release kinetics by modification of the existing intermolecular interactions (see below). 22,23,25,28,41,42 Moreover, EE values were barely constant for the derivatized CNPs independently of the fed siRNA amount, in contrast to nonderivatized ones for which a maximum $\mathrm{EE}$ was found at 150 pmol.

It is also worth mentioning that similar derivatized chitosans were previously used to entrap the protein insulin in CNPs. ${ }^{25}$ In that study, maximum values of $\mathrm{EE}$ and $\mathrm{LC}$ were attained for NPs consisting of derivatized chitosan with a degree of 
substitution of $10 \%$, whereas those of $50 \%$ showed the lowest values. Possibly, the competitiveness between protein complexation and compaction of the hydrophobic side chains in the NP core might explain that outcome. Nevertheless, neither the length of the aliphatic side chains nor the substitution degree of the chitosans forming the NPs showed statistically significant differences in both the values of LC and EE for siRNA encapsulation.

On the other hand, a gel electrophoresis assay additionally confirmed the siRNA complexation into CSNPs. The concentration of CSNPs was selected to get a final siRNA concentration of $90 \mathrm{nM}$ per well. As observed in Figure S3, free siRNA (lane 1) exhibited a trailing band usually associated with siRNA degradation, and no fluorescence was observed in the associated well. Nevertheless, for the remaining lanes, a strong fluorescence was observed indicating that siRNA was inside the CSNPs, and traces of nonencapsulated siRNA were washed away after the cleaning steps (see Section 2 for details).

In addition, NP sizes and $\zeta$-potentials of the different types of CSNPs initially loaded with $150 \mathrm{pmol}$ of siRNA were determined by DLS and electrophoretic mobility analysis, respectively (see Figure 4). CSNPs with a feed of 150 pmol were chosen for further studies because they showed a suitable balance between the initial fed concentration and high EE and LC values. As depicted in Figure 4a, nonderivatized CSNPs showed, in general, bulkier sizes than their empty counterparts (CNPs), ${ }^{43}$ with this trend being strikingly different when the NPs were formulated with derivatized chitosan. As previously discussed, this might be a consequence of the compaction of NP cores to avoid the exposure of their aliphatic side chains to the aqueous medium, thus promoting collective interactions inside the particles, particularly with entrapped siRNA molecules. Conversely and as noted for CSNPs with chitosans modified with a 12-carbon side chain, the excluded volume effect of polymer chains might have precluded an effective NP packing leading to an NP size enlargement. Based on the aforementioned results, one might see some competition between cargo complexation and the compaction of the chitosan hydrophobic side chains in the NP cores, which definitively controlled the overall characteristics of the NPs. ${ }^{44,45}$

TEM images would also support our previous view. Figure $4 \mathrm{c}, \mathrm{d}$ shows that derivatized $8 \mathrm{Mb}$-CSNPs displayed a smooth outer shell suggesting that side chains were located inside (see Scheme 2). Conversely, $12 \mathrm{Mc}$-CSNPs showed a hairy shell indicating that some segments of the side chains were located at the NPs' surfaces.

This picture was also corroborated by $\zeta$-potential data. $\zeta$ Potentials for all CNPs and CSNPs tested were relatively high and positive (Figure 4b), which is expected to result in a good colloidal stability (see below for details). As mentioned previously, such values roughly decreased with both the molecular weight and substitution degree of the chitosan backbone. As opposed to bare NPs, modified NPs possess roughly constant $\zeta$-potential values regardless of the graft density and side-chain length. These observations would, hence, support DLS data and our hypothesis that hydrophobic side chains might have localized themselves inward, avoiding contact with water through entanglements with the rigid siRNA and, at the same time, displacing the charged amine groups of chitosan to the NP outer shell (see Scheme 1). As depicted by ITC data (see above) and inferred by others, $^{28,46,47}$ hydrophobic interactions inside NPs seem to play an important role in the complexation process, which further supports our previous hypothesis. ${ }^{25}$

3.4. Colloidal Stability of CSNPs. Suitable size distributions and colloidal stability of CSNPs are crucial factors for their successful biopharmaceutical application. For example, the evolution of the particle size affects not only endocytosis by tumoral cells but also the NPs' ability to evade recognition and clearance by the reticuloendothelial system. Hence, we next analyzed the temporal evolution of particle size and $\zeta$-potentials under different conditions as suitable indicators of the colloidal stability of the as-obtained CSNPs.

Upon incubation for 3 days in water at $37^{\circ} \mathrm{C}$, $\zeta$-potentials of derivatized CSNPs remained almost unchanged (Figure 5a). In addition, the size temporal evolution of unmodified and derivatized CSNPs analyzed by DLS for 5 days in aqueous solution at $37{ }^{\circ} \mathrm{C}$ (Figure $5 \mathrm{~b}-\mathrm{d}$ ) denoted that 8- and 12carbon derivatized CSNPs were very stable in water, as shown by the barely constant particle sizes, during the whole incubation period. It can be observed that 12-carbon derivatized CSNPs displayed again larger sizes in water compared to 8-carbon hydrophobized ones since the side chains would be not completely packed into the particle core as they are exposed to the solvent as previously mentioned. Therefore, this may be considered an additional clue of the exposure of longer side chains near/on the particles surfaces as a result of their inefficient packing inside the particle cores. For the NPs, containing unmodified chitosans, a certain size decrease was observed, during the first $24 \mathrm{~h}$, which could be related to some siRNA release from the particles and/or partial hydrolysis induced by the scission of polymeric chains. ${ }^{48}$

Conversely, in DMEM supplemented with $10 \%$ (v/v), FBS particle sizes were observed to continuously rise for the first 48 $\mathrm{h}$ of incubation and then remained barely constant. Such increases may arise from the formation of aggregates among the CSNPs (from 3 to 6 units, in terms of a single NP size) owing to protein adsorption onto the NP surfaces, particularly serum albumin, through electrostatic and hydrophobic interactions, giving rise to a protein biocorona in a process known as opsonization. ${ }^{49}$ Together with this, some NP agglomeration, provoked by solution ionic strength, may occur. $^{50}$ Interestingly, it could be observed that CSNPs based on the 12-carbon derivative of chitosan displayed smaller size increments compared to those of 8-carbon derivatives. This could be explained based on the existence of larger electrostatic attractions between the deprotonated carboxylic acids of proteins in FBS and the protonated amines of chitosan at the surface of these NPs. Besides, we also think that the 12-carbon side chains of chitosan, supposedly being exposed near/on the particle surfaces, might somehow hinder protein adsorption to a certain extent, thus avoiding further size increments. ${ }^{51,52}$ However, additional studies would be further required to confirm this point. After $48 \mathrm{~h}$ of incubation, 8-carbon hydrophobized CSNPs did not lead to substantial changes in particle sizes, whereas for 12-carbon derivatized ones, a certain decrease was observed. Finally, the stability of the different CSNPs at different solution $\mathrm{pH}$ values for 5 days was also monitored by DLS. The different types of CSNPs showed almost no changes in particle sizes upon incubation, except at the most extreme $\mathrm{pH}$ values of 3.0 and 8.0 (see Figure S4 and further information in SI).

3.5. Stability of Encapsulated siRNA. For effective gene expression and/or inhibition, nucleic acids or oligonucleotides entrapped in a delivery vehicle should be protected from 

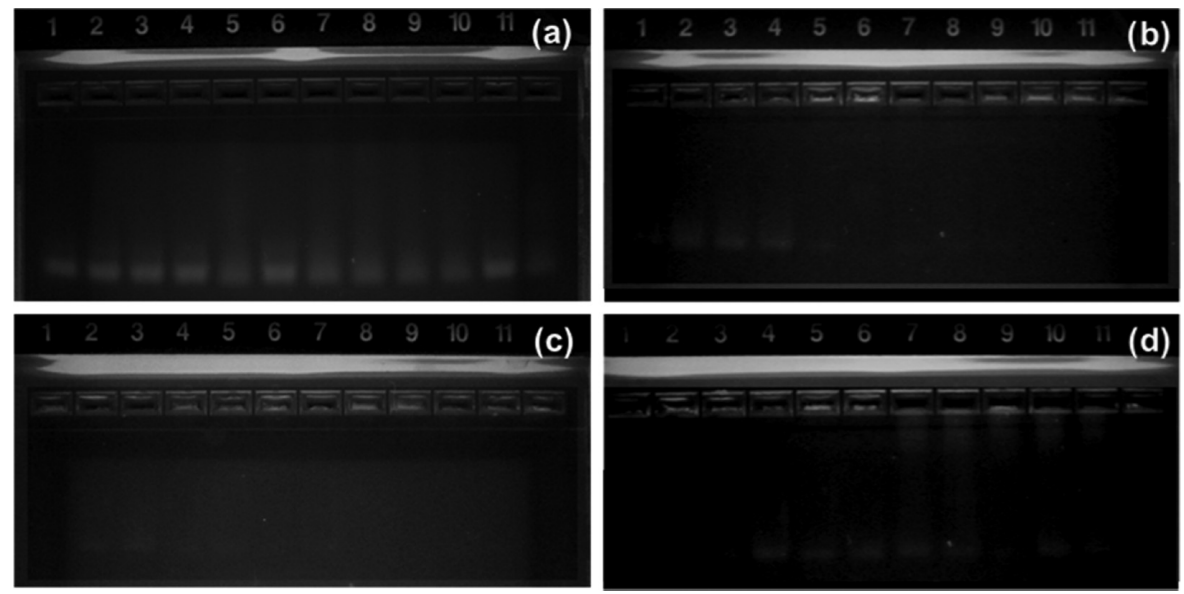

Figure 6. Mobility of siRNA following incubation in FBS-enriched medium: (a) naked siRNA; CSNPs composed of (b) CM, (c) 8Mb, and (d) $12 \mathrm{Mc}$. From lane 1 to 6 , incubation was done in 5\% (v/v) FBS; incubation in lanes 7-12 was done in 50\% (v/v) FBS. Incubation times were as follows: 0 min (lanes 1 and 7), 30 min (lanes 2 and 8), $2 \mathrm{~h}$ (lanes 3 and 9), $7 \mathrm{~h} \mathrm{(lanes} 4$ and 10), $24 \mathrm{~h} \mathrm{(lanes} 5$ and 11), and $48 \mathrm{~h}$ (lanes 6 and 12).
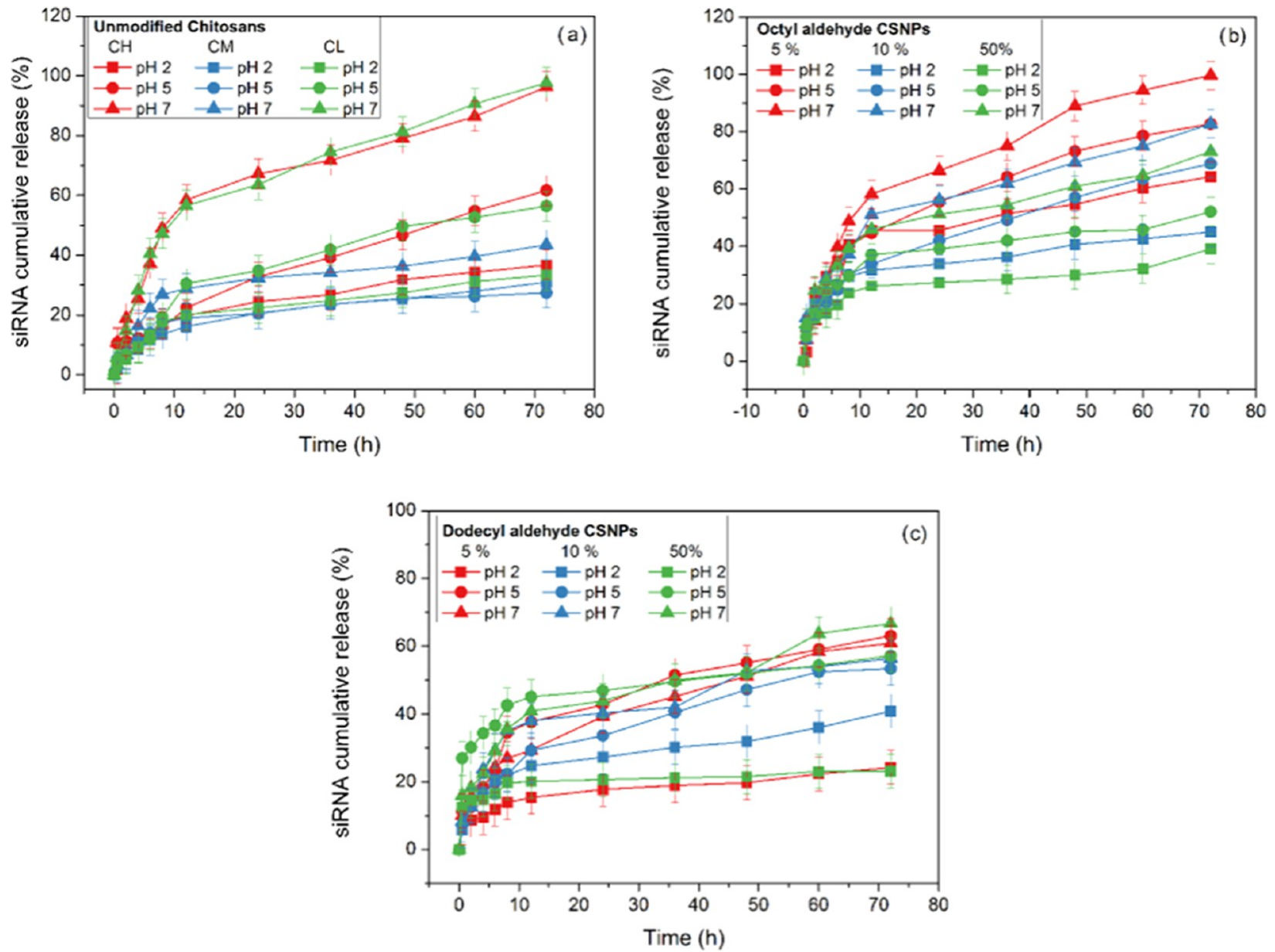

Figure 7. SiRNA release from CSNPs at pH values of $7.4(\boldsymbol{\Delta}), 5.5(\mathbf{\theta})$, and $2.0(\boldsymbol{\square})$. (a) NPs containing unmodified chitosans with (red) high, (blue) medium, (green) low molecular weight. (b) 8-Carbon (c) and 12-carbon side-chain-modified CSNPs with (red) 5\%, (blue) 10\%, and (green) $50 \%$ of substitution degree.

degradation by enzymes such as serum nucleases. ${ }^{53,54}$ In the present case, if the CSNPs were unstable, siRNA would be released. ${ }^{37}$ Then, we analyzed the influence of substitution degree and side-chain length on the NP-entrapped siRNA stability by selecting two extreme cases: a modified chitosan with a short aliphatic chain and a low substitution degree
$(\mathrm{C} 8 \mathrm{Mb})$ and another modified chitosan with a longer side chain and a much higher substitution degree (C12Mc). To do that, an electrophoresis gel assay after particle incubation was performed in DMEM supplemented with 5 and $50 \%(\mathrm{v} / \mathrm{v})$ FBS at $37^{\circ} \mathrm{C}$ to ensure the ability of these modified CSNPs to 
protect siRNA from digestion by serum nucleases (see Section 2 for details).

As observed in Figure 6a, naked siRNA was intact only at the beginning of incubation in 5\% (v/v) FBS (no trailing band was observed in lane 1 at $0 \mathrm{~min}$ ) and fully degraded at $24 \mathrm{~h}$. Conversely, at higher serum concentration, $50 \%$ (v/v) FBS, the presence of a trailing band in all lanes was indicative of instant degradation, in agreement with previous reports. ${ }^{10}$

When unmodified CSNPs were incubated in 5\% (v/v) FBS, siRNA started to degrade after $30 \mathrm{~min}$ of incubation (no trailing band was observed in lane 1), whereas full degradation was observed only after $24 \mathrm{~h}$ (Figure 6b). Conversely, siRNA recovered from $\mathrm{C} 8 \mathrm{Mb}$ - and $\mathrm{C} 12 \mathrm{Mc}$-derivatized CSNPs was intact after $7 \mathrm{~h}$ of incubation and largely complexed to the former type of NPs and fully degraded for the latter after $48 \mathrm{~h}$ of incubation (Figure $6 c, d$ ). siRNA loaded in CSNPs with the $\mathrm{C} 12 \mathrm{Mc}$ derivative of chitosan manifested a similar behavior as that of naked siRNA in $50 \%(\mathrm{v} / \mathrm{v})$ FBS, that is, the presence of a trailing band in all lanes (Figure 6d, lanes 7-12). This observation might support our hypothesis that for C12Mcderivatized CSNPs, siRNA would be entrapped especially around the particle surfaces as it is largely exposed to nucleases. Surprisingly, C8Mb-derivatized CSNPs seemed to preclude the contact of siRNA with serum (see Figure 6c); that is, a great part of siRNA would be still complexed with the modified chitosan chains inside the NPs, and only a small detachment was observed in the presence of heparin, as confirmed by the lowest signals in the electrophoretic lanes. Therefore, it is concluded that the present C8Mb-CSNPs significantly protected siRNA from nuclease activity, which may hold promise as suitable gene-delivery nanovectors.

3.6. siRNA Release. As previously mentioned, a good NP stability is crucial for extracellular siRNA protection; however, a suitable particle disassembly process is also needed to allow cargo delivery and subsequent RNA-mediated gene silencing through interactions with intracellular components such as the RNA-induced silencing complex (RISC). Then, an appropriate balance needs to be achieved between siRNA protection and release for therapeutic efficacy. To this end, the ability of CSNPs to release the entrapped siRNA was further measured at $37{ }^{\circ} \mathrm{C}$ for several days at three different $\mathrm{pH}$ values mimicking different biological media. Herein, CSNPs were incubated at $\mathrm{pH}$ 7.4, 5.5, and 2.0, which mimick the physiological $\mathrm{pH}$ for parenteral administration, the acidic environment of tumors and some intracellular compartments like lysosomes, and the gastric environment, respectively. ${ }^{34}$

As observed in Figure 7, siRNA release from CSNPs was characterized by an initial fast release (burst phase) within the first $9-13 \mathrm{~h}$ of incubation, in which the amount of cargo released may correspond to siRNA located on/near the NP surfaces. The burst phase was followed by a quasilinear region leading to a sustained siRNA release for several days, as previously observed, for example, for hydrophobic glycol chitosan-based NPs. ${ }^{55}$ For the treatment of many different diseases, a sustained release pattern is desired to maintain a constant cargo concentration in blood and to prevent its premature leakage, which can lead to undesired toxic side effects. An additional look at the obtained release profiles shows that the derivatization of chitosans and subsequent NP formation allowed us to perfectly tune release rates and extents; that is, for a given condition, there is a CSNP with a suitable release profile. In this manner, the in vitro release behavior of siRNA was observed to be dependent on both the hydrophobicity of the chitosan biopolymer and the $\mathrm{pH}$ of the release medium, especially within the burst region. Briefly, it is necessary to remind that cargoes encapsulated in biodegradable polymeric matrices/NPs are released by three mechanisms taking place in sequence: (i) desorption from the particle surface, (ii) diffusion and readsorption through the pores of the polymeric network, and (iii) degradation and erosion of the polymeric network. Also, solution $\mathrm{pH}$ may have affected the $\zeta$-potential values and sizes (see Figure S4, for example) of the polymeric NPs. ${ }^{25}$ For example, CSNPs made of CM had a $\zeta$-potential of $25.4 \pm 1.0 \mathrm{mV}$ at $\mathrm{pH} 5.0,5.1 \pm 0.8 \mathrm{mV}$ at $\mathrm{pH}$ 7.0, and $52.2 \pm 1.4 \mathrm{mV}$ at $\mathrm{pH}$ 3.0. Conversely, for CSNPs using $\mathrm{C} 12 \mathrm{Mc}$, the $\zeta$-potentials obtained were $22.7 \pm 1.1,35.2$ \pm 1.1 , and $48.3 \pm 2.7 \mathrm{mV}$ at $\mathrm{pH}$ values of 3.0, 5.0, and 7.0, respectively. The extent of siRNA release from the different derivatized and nonderivatized CSNPs generally followed the sequence $\mathrm{pH} 7.4>\mathrm{pH} 5.5>\mathrm{pH}$ 2.0. Therefore, when the solution $\mathrm{pH}$ is larger than the $\mathrm{p} K_{\mathrm{a}}$ of TPP and siRNA, CSNPs are highly negatively charged and inner electrostatic repulsions should facilitate a faster siRNA release. At $\mathrm{pH}$ 5.5, such forces were diminished and cargo molecules would be released by simple diffusion. At $\mathrm{pH} 2.0$, the chitosan amine groups were entirely protonated and the cargo release rate was much lower. In this case, siRNA might have acted as an additional "crosslinker", thanks to the electrostatic attractions with the backbone and $\mathrm{NH}_{3}^{+}$groups of chitosan. ${ }^{10,56,57}$

The observed release profiles denoted a certain dependence on the chitosan molecular weight (Figure 7a). In particular, CM-based CSNPs exhibited a more sustained release than CLand $\mathrm{CH}$-based ones. This observation could be explained on the basis that CL-based CSNPs might not have completely compacted siRNA into stable particles, allowing a faster release. ${ }^{58,59}$ Meanwhile, CH-based CSNPs would have a lower cross-linking density than CM-based ones (larger chitosan/ TPP ratio), thus inducing the formation of a less compact and more porous polymeric network. ${ }^{60}$

The hydrophobization of chitosan chains also had a clear influence on the siRNA release rates, where both the side-chain length and substitution degree played an important role. Li et al. analyzed the permeation and diffusion of encapsulated vitamin B2 within unmodified and alkylated CNPs at different $\mathrm{pH}$ values. ${ }^{61}$ These authors found that drug release from unmodified CNPs increased when raising the solution $\mathrm{pH}$ as a consequence of the dissociation and reconstruction of hydrogen bonding. In basic medium, the dissociation of hydrogen bonds lowered the intermolecular interaction force between the side chains and the polymeric network, rendering it loose and more porous and, thus, the drug diffused out more easily. Conversely, for alkylated CNPs, drug permeation and diffusion decreased as the solution $\mathrm{pH}$ increased. In acidic medium, both permeation and diffusion diminished with the increase in the alkyl side-chain length, whereas the opposite behavior was found under basic conditions. This observation is in agreement with the results shown here, where C8-modified CSNPs displayed, in general, faster release profiles than C12modified ones under acidic conditions, whereas the opposite trend was found at $\mathrm{pH}$ 7.4. The elongation of the side chains increased the hydrophobicity of the polymeric network within the NPs, which, in turn, hindered the outward diffusion of siRNA through the NP matrix. Under basic conditions, an enhancement of diffusion was noted as the number of carbon atoms in the side chain increased, likely related to a larger steric hindrance and excluded volume effects. The extent of 

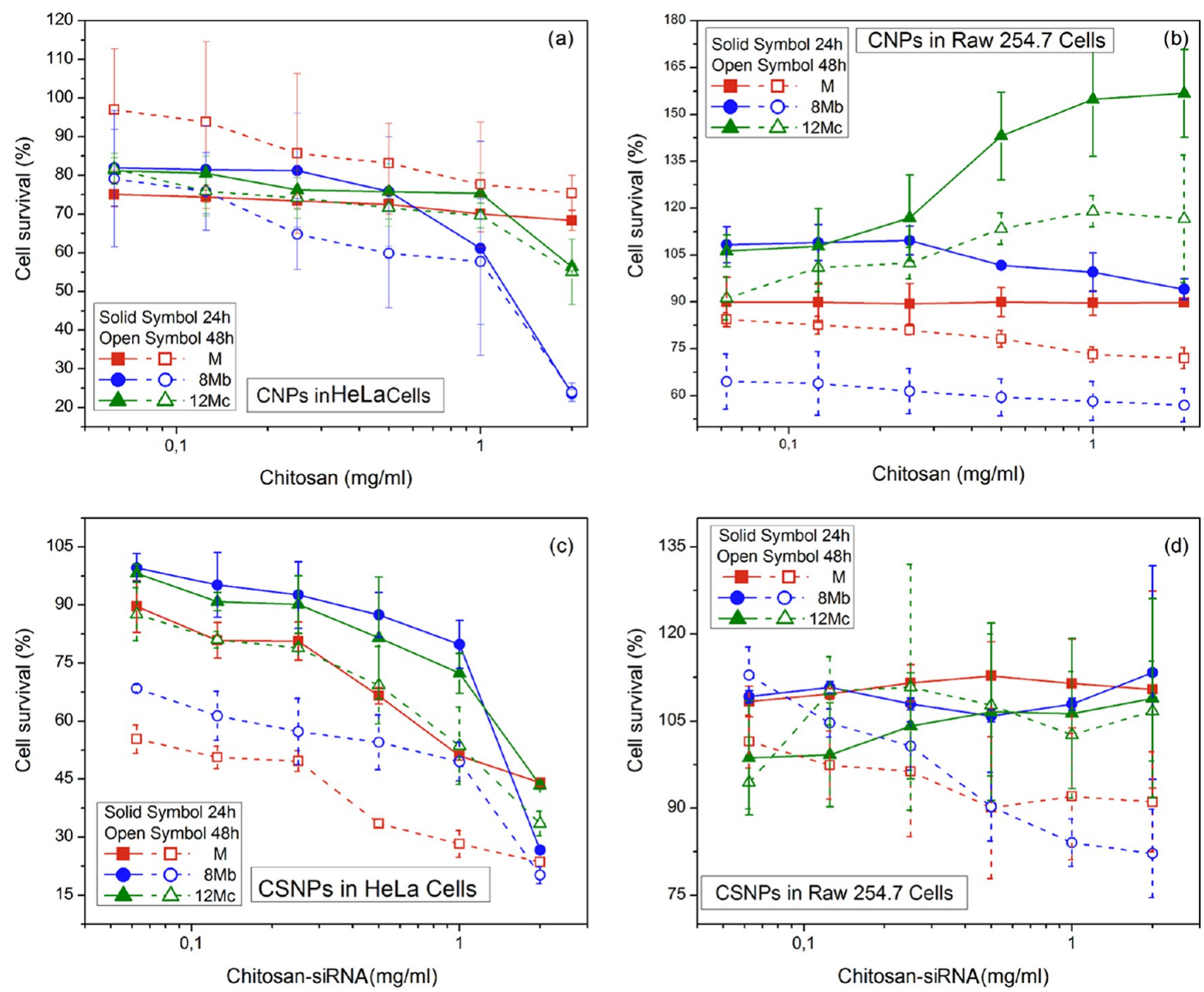

Figure 8. Cell survivals for (a, b) CNPs and (c, d) CSNPs in (a, c) HeLa cells and (b, d) macrophages. Solid symbols represent incubation for 24 h, whereas open symbols for $48 \mathrm{~h}$. $(\boldsymbol{\square}, \square)$ Medium-molecular-weight chitosan, $(\boldsymbol{\bullet}, \bigcirc) 8 \mathrm{Mb}$, and $(\boldsymbol{\Delta}, \triangle) 12 \mathrm{Mc}$.

siRNA release was reduced as the substitution degree in the chitosan backbone increased as a consequence of the larger density of the particle core, eventually restricting the siRNA diffusion out of the particles.

3.7. In Vitro Cell Biocompatibility. We next evaluated the potential cytotoxicity of both CNPs and CSNPs after cell $\mathrm{NP}$ uptake and internalization by measuring cell metabolic activities via the CCK-8 proliferation assay in two different cell lines, human cervical cancerous HeLa and mice RAW 264.7 macrophage cells. Different variables such as NP concentration, exposure time, and the nature of the NP (hydrophobicity) were analyzed. HeLa cells were chosen since they represent a classical immortalized tumoral cell line to test in vitro the efficacy of new drugs/formulations for cancer treatment. Macrophages are one of the principal immune effector cells that play essential roles as secretory, phagocytic, and antigen-presenting cells in the immune system. We chose RAW 264.7 macrophage cells since they also display a high sensitivity to potentially toxic chemicals/compounds. ${ }^{6-64}$

$\mathrm{C} 8 \mathrm{Mb}$ - and $\mathrm{C} 12 \mathrm{Mc}$-based CSNPs were selected as examples of extreme derivatizations ( $8 \mathrm{Mb}$ NPs possess short side chains and low substitution degree, whereas $12 \mathrm{Mc}$ NPs have longer side chains and much higher substitution degree), and also showed some of the best physicochemical properties in terms of particle sizes and surface charges, siRNA protection, serum stability, and sustained release profiles. In addition, CM-based CSNPs were selected as controls for comparison.

Figure 8 shows the cell viabilities of the selected CNPs and CSNPs in both HeLa and RAW 264.7 cell lines after 24 and 48 $\mathrm{h}$ of incubation (see also Figure S5 in SI for additional data). As observed in this figure, HeLa cells were more sensitive than macrophages to the presence of CNPs and CSNPs, in agreement with previous observations. ${ }^{65}$ In HeLa cells, cell viability was dose dependent for CNPs and, especially, for CSNPs. Such dependence is not particularly observed in macrophages, except for the highest particle concentrations tested. ${ }^{17,66}$ Nevertheless, the present biopolymeric NPs were observed to be nontoxic to cells, with viabilities always above $50 \%$, except at the highest concentration $(2 \mathrm{mg} / \mathrm{mL})$ after $48 \mathrm{~h}$ of incubation. ${ }^{67}$ In general, it was also observed that cell toxicity was relatively larger at longer incubation times. Moreover, the incorporation of siRNA into the CSNPs rendered them less toxic to cells than the empty ones (CNPs), especially in macrophages. 
It was also noted that differences in survival rates were also cell-type dependent. In nonphagocytic cells such as HeLa, cationic surface charges and small sizes for most NPs correlated with higher cellular uptakes (see below) and greater cell cytotoxicities. Conversely, phagocytes such as macrophages and monocytes are more sensitive to the presence of bigger and negatively charged NPs. The presence of serum appeared to reduce NP uptake in nonphagocytic cells but increase NP uptake in phagocytic ones. ${ }^{68,69}$ For HeLa cells, it was found that $\mathrm{C} 8 \mathrm{Mb}$ and $\mathrm{CM}$ CNPs have similar $\zeta$-potentials, but $\mathrm{C} 8 \mathrm{Mb}$ particles exhibited lower survival rates at $48 \mathrm{~h}$ of incubation. For RAW 264.7 cells, the opposite trend was found. Surprisingly, when siRNA was incorporated inside the derivatized CSNPs, higher survival rates in both kinds of cell lines were detected (see Figure S5 in SI). Importantly, CSNPs with a 12-carbon alkyl chain chitosan derivative achieved higher biocompatibility compared to the 8-carbon-based ones and, generally, cell toxicity progressively decreased upon increasing the level of substitution of the polymeric backbone when compared to their unmodified homologues (see Figure S5 in SI). The reason for such behavior is still unclear, but it might be related to the enhanced hydrophobicity of the derivatized CSNPs.

To further elucidate the influence of the type and extent of chitosan hydrophobization on cell viability, relative cell toxicities $(\Delta)$ were calculated. $\Delta$ is defined as the difference in cell survival in the presence of CNPs and CSNPs. $\Delta>0$ indicated that CSNPs induced lower cellular survival rates than CNPs, whereas $\Delta<0$ indicated the opposite behavior. Chitosan hydrophobization effectively displayed a positive effect on cell viability, and the type and extent of modification played a key role. It was observed that CSNPs were more biocompatible than CNPs, except in HeLa cells after $48 \mathrm{~h}$ of incubation (see Figure S6 in SI).

3.8. Cellular Uptake and Transfection Efficiency. To analyze the in vitro cellular uptake and transfection efficiency of CSNPs, fluorescence microscopy and flow cytometry experiments were carried out. A FITC-labeled scrambled RNA oligo was used as cargo to effectively confirm the particle internalization and cargo release inside the cell cytoplasms. CSNPs produced from $\mathrm{C} 8 \mathrm{Mb}$ and $\mathrm{C} 12 \mathrm{Mc}$ derivatives of chitosan were tested as optimal siRNA carriers, whereas the CM-based ones were used as a reference. Figures 9 and S7 show the temporal evolution of particle internalization inside HeLa and RAW 264.7 cells, respectively. Cellular uptake was monitored in macrophages for 2 and $6 \mathrm{~h}$, whereas in HeLa cells, lapse times of 6,12 , and $24 \mathrm{~h}$ were used. The choice of different time points was based on the strong phagocytic activity of macrophages, which rapidly engulf a wide variety of foreign agents such as pathogens, particles, apoptotic cells, constituting a defense mechanism of the host. ${ }^{70}$

Figure 9 depicts a progressive particle accumulation and subsequent siRNA release as shown by the enhancement of the green fluorescence from the dye-labeled oligo throughout incubation. Interestingly, CSNPs formulated with derivatized chitosan resulted in a progressive accumulation of siRNA in HeLa cells, where the fluorescence pattern turned red to orange-green in the merged images. However, the CSNPs bearing unmodified chitosan displayed a less intense fluorescent signal, which could be ascribed to the lower amount of siRNA loaded, as compared to those with modified chitosan. For C8Mb- and C12Mc-CSNPs, the fluorescence was rather intense after $6 \mathrm{~h}$ of incubation, in agreement with their

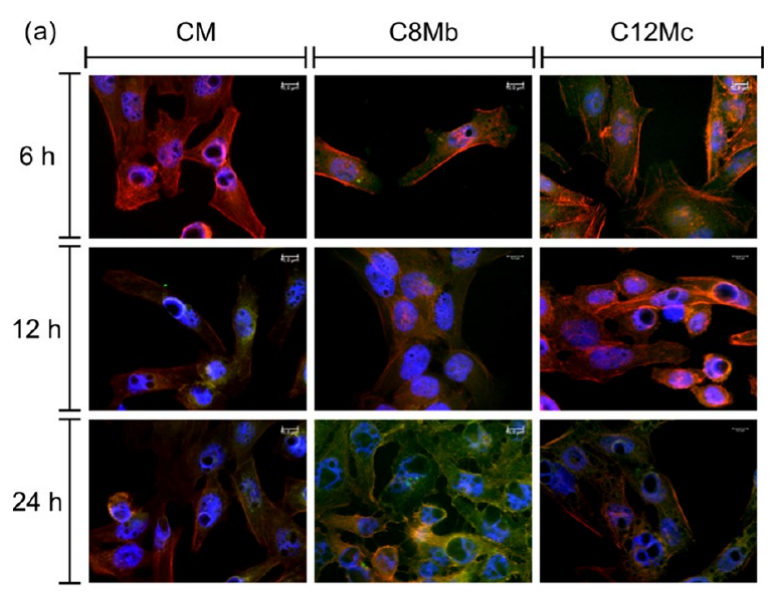

(b)

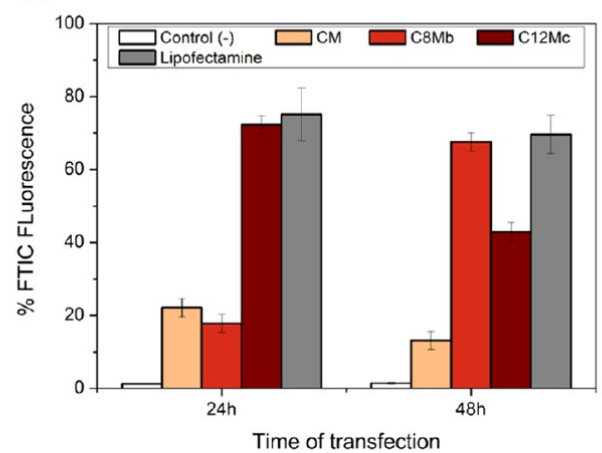

Figure 9. (a) Fluorescence microscopy images of cellular uptake and intracellular distribution of FITC-siRNA (green channel) in HeLa cells for 6,12 , and $24 \mathrm{~h}$. The blue fluorescence stemmed from cell nuclei stained with DAPI and the red fluorescence from the cell cytoplasm stained with BODIPY Phalloidin. Microphotographs were presented as merged images to facilitate their understanding. Scale bars are $10 \mu \mathrm{m}$. (b) Transfection efficiency of FTIC-siRNA in HeLa cells measured by flow cytometry using $\mathrm{CM}-, \mathrm{C} 8 \mathrm{Mb}$-, and $\mathrm{C} 12 \mathrm{Mc}-$ CSNPs. Nontransfected HeLa cells were used as a negative control, whereas siRNA transfection with Lipofectamine 2000 was used as the positive one.

slightly larger cargo release rates at short incubation times (see Figure 7). However, the fluorescence signal after $12 \mathrm{~h}$ for C12Mc-CSNPs was surprisingly diminished, whereas at $24 \mathrm{~h}$, it was recovered. These findings would support our hypothesis regarding the initial siRNA release from the outermost shell of the C12Mc-CSNPs shortly after incubation, followed by its slow diffusion from the NPs' core (see the discussion below). To additionally confirm that siRNA was released inside the cells, three-dimensional (3D) reconstructions of $z$-stack images in both kinds of cells were performed. The reconstructed images (Figure S8 in SI) allowed us to confirm that siRNA was effectively delivered to and diffused along the cell cytoplasm.

Similarly, NP accumulation inside RAW 264.7 macrophages increased over time, namely, for CM-CSNPs, which manifested the highest cell uptake as opposed to the observations in HeLa cells. The elevated levels of toxicity of CM-CSNPs, previously observed, may correlate to their higher cell uptake (Figure S7). Moreover, the observed fluorescence intensity was lower for $\mathrm{C} 8 \mathrm{Mb}$-CSNPs than for $\mathrm{C} 12 \mathrm{Mc}$-ones. Also, the resulting fluorescence pattern visualized for C12Mc-CSNPs appeared mostly punctuated, suggesting particle aggregation.

Finally, a quantitative analysis of the particle uptake and subsequent transfection in terms of the percentage of FITC 
fluorescence inside HeLa cells was also performed by means of flow cytometry. Figure 9b shows that cells transfected with CM-CSNPs displayed a low fluorescence during the selected incubation period. This could be a result of the lower LCs attained as well as the slower release rates observed for this class of NPs in solutions of pH 5.5-7.4 (see Table S2 and Figure 7a). Moreover, cells transfected with C8Mb-CSNPs displayed a rather similar fluorescence signal, ca. $20 \%$, as those transfected with CM-CSNPs after $24 \mathrm{~h}$ of incubation; conversely, at $48 \mathrm{~h}$, the extent of transfection largely increased up to $70 \%$, similar to that of Lipofectamine 2000 used as a positive control. Finally, C12Mc-CSNPs achieved comparable levels of transfection with the control at $24 \mathrm{~h}$, which substantially declined after $48 \mathrm{~h}$ of transfection. This behavior can be a consequence of a stronger interaction of this type of NPs with cell membranes, facilitating a faster particle cell uptake as well as a slightly quicker cargo release compared to those of C8Mb-based and unmodified ones (see Figure 7).

Indeed, it has been already suggested that hydrophobic side units in polymeric nanocarriers may increase the transfection efficiency through modulation of NP interactions with cells, enhancing their adsorption on the cellular surface and subsequent uptake. ${ }^{58,71,72}$ Also, hydrophobic segments in polymeric nanocarriers may help in the disassembly of chitosan/DNA and chitosan-siRNA NPs to facilitate the release of the genetic material that would, otherwise, be strongly bound through ionic interactions to the polymeric backbone. Hence, such favorable characteristics of the hydrophobic side chains, grafted onto chitosan, may account for the prominent transfection efficiencies obtained in this work when compared to the unmodified biopolymer. In this regard, Liu et al. prepared alkylated chitosans and observed that the transfection efficiency in a mouse $\mathrm{C} 2 \mathrm{C} 12$ cell line ( $\mathrm{C} 3 \mathrm{H}$ muscle myoblast) improved with an increase in the length of the substituted side chain up to eight carbons, which is in fair agreement with our data. ${ }^{22}$ Also, Chae et al. synthesized deoxycholic-acid-modified chitosans as the main component of nanocarriers, for pEGFP-N1 plasmid DNA delivery, achieving a more pronounced transfection efficiency in HEK 293 cells, which was dependent on the degree of susbstitution. ${ }^{73}$ Similarly, Hu et al. grafted stearic acid onto the chitosan backbone to transfect the pDNA pEGFP-C1 into A549 cells, obtaining larger transfection efficiencies with much lower cell mortalities. $^{74}$

3.9. Efficiency of siRNA Gene Silencing. Finally, to analyze the silencing activity of released siRNA from the selected derivatized chitosan-based NPs, we monitored the fluorescence intensity decrease induced by a specific siRNA against the expression of GFP transfected in modified HeLa cells using CM-, C8Mb, and C12Mb-CSNPs by means of fluorescence microscopy and flow cytometry. Standard HeLa cells as a negative control and nontransfected GFP-HeLa cells as a positive one were used. siRNA transfected with Lipofectamine 2000 was a secondary positive reference for comparison. According to the qualitative analysis by fluorescence microscopy, depicted in Figure 10a, the intrinsic fluorescence of cells was almost maintained following transfection up to $6 \mathrm{~h}$, after which its intensity gradually diminished. Nonetheless, a significant portion of fluorescence $24 \mathrm{~h}$ posttransfection was detected, which could be assigned to (i) the sustained release of siRNA molecules inside the cytoplasm and/or (ii) an insufficient time to achieve remarkable inhibition of protein expression. The GFP inhibition using

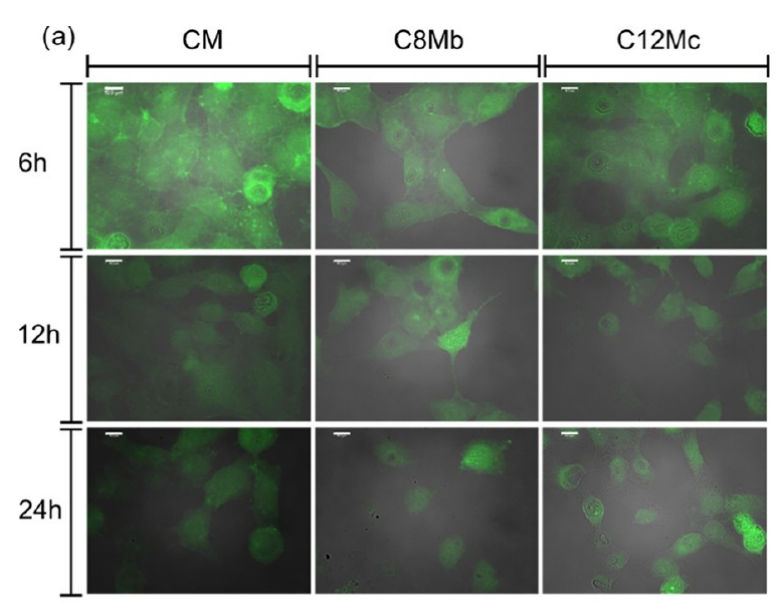

(b)

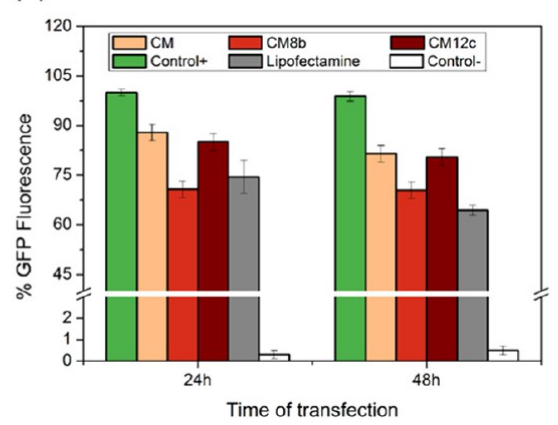

Figure 10. (a) Gene silencing observed by fluorescence microscopy in GFP-HeLa cells exposed to chitosan-based NPs loaded with an siRNA against GFP. Images were taken at 6,12 , and $24 \mathrm{~h}$ of incubation. Chitosans $\mathrm{CM}, \mathrm{C} 8 \mathrm{Mb}$, and $\mathrm{C} 12 \mathrm{Mc}$ were used. Merged images are shown. Scale bars are $10 \mu \mathrm{m}$. (b) Mean fluorescence intensity of GFP expressed in HeLa cells after 24 and $48 \mathrm{~h}$ of transfection with anti-GFP siRNA using CM, C8Mb, C12Mc-CSNPs, and Lipofectamine 2000.

$\mathrm{C} 8 \mathrm{Mb}$ and $\mathrm{C} 12 \mathrm{Mb}-\mathrm{CSNPs}$ seemed to be rather larger than that of CM-CSNPs probably as a consequence of (i) a lower LC, (ii) a slower release rate, and (iii) a stronger chitosansiRNA interaction, which may prevent siRNA dissociation from the former NPs. ${ }^{72,75}$

Quantitative analysis of GFP knockdown efficiency was further conducted in the same cell line by flow cytometry using CSNPs with the modified and nonmodified chitosans. Here, it is necessary to remind that a suitable balance between siRNA protection and efficient siRNA release must be achieved to obtain simultaneously high levels of transfection and silencing. Figure $10 \mathrm{~b}$ shows that $\mathrm{C} 8 \mathrm{Mb}-\mathrm{CSNPs}$ exhibited the greatest gene-silencing activity, ca. $30 \%$, at both 24 and $48 \mathrm{~h}$ of incubation and similar to that of the Lipofectamine 2000 positive control. In contrast, the CM-CSNPs suppressed GFP expression by $10-15 \%$, which can be rationalized on the basis on their lower LC values, slow release rates, and serum instability, as described previously. Surprisingly, rather similar low knockdown efficiencies (ca. 20\%) were also noted for C12Mc-CSNPs despite their much higher LC and particle uptake. It could be argued that these CSNPs might have undergone larger siRNA degradation by nucleases, as confirmed by gel electrophoresis (Figure 6d), probably due to the presence of some siRNA in the outermost shell of their surfaces, as previously hypothesized (see Scheme 1). In this regard, low silencing activities were also observed for particles prepared by simple complexation of siRNA onto preformed 
chitosan-TPP NPs as a consequence of the inefficient protection of the genetic material to enzymatic degradation. ${ }^{10,76}$ Therefore, in spite of the greater transfection of the C12Mc-NPs than that of C8Mb-NPs, after 24 h (see Figure 9), the potential cleavage of siRNA released from the former ones might have impaired their gene-silencing performance.

\section{CONCLUSIONS}

In summary, this work entailed hydrophobic modification of chitosan, prior to its use in NP production via ionotropic gelation, for siRNA delivery. The structural modifications introduced had an impact on siRNA loading capacity and release patterns, as well as on its transfection efficiency and gene knockdown activity. This was pursued by the alkylation procedure, where 8,10 , and 12 -carbon side chains were grafted onto the chitosan backbone, at different substitution degrees $(5,10$, and $50 \%)$. The obtained experimental data indicated that the side-chain length played a key role in determining particle sizes and surface charges, especially at high substitution degrees, through controlling the nature and extent of interactions between siRNA and the modified chitosan backbone, as shown by ITC data. Likewise, the incorporation of siRNA into the NPs further induced significant changes in their surface charges as well as sizes, becoming smaller, due to the compaction of the NP cores. Moreover, enhanced siRNA $\mathrm{EE}$ and LC values were obtained for NPs formulated with the hydrophobically modified chitosans compared to those of their homologues prepared with the unmodified chitosan. Besides, TEM images enabled to view the diverse morphologies of the NPs produced, where, for instance, the C8M-CSNPs displayed a smooth surface, whereas the C12M-CSNPs showed a hairylike structure. This may justify the alteration in the siRNA release profiles among CSNPs, with the hydrophobically modified and unmodified chitosan. Concerning stability against nucleases, chitosans of higher degrees of substitution and/or longer side chains ( $>8$ carbons) provided lower protection to siRNA, supposedly because a part of their side chains was displaced to the surface, and with some siRNA excluded outward, making it more prone to enzymatic degradation. Nevertheless, further analyses are warranted to ascertain this premise and would be revealed in a forthcoming publication.

On the other hand, hydrophobically modified and base CSNPs were, in general, nontoxic to HeLa and RAW 264.7 cells, the former showing a dose-dependent response. Similarly, cell toxicity and uptake were also found to be cell dependent. Cell internalization and transfection efficiencies were observed to be larger for the hydrophobically modified CSNPs, with slightly better GFP knockdown efficiencies, for example, for C8Mb-CSNPs compared to those for Lipofectamine 2000 control. This observation probably originated from the particular architecture of this type of CSNPs, where siRNA was tightly packed in their cores. However, in the case of C12Mc-CSNPs, for example, a proportion of siRNA adsorbed onto the surfaces, probably by the excluded volume effect, thus impeding its protection against degradation, as corroborated by gel electrophoresis.

\section{ASSOCIATED CONTENT}

\section{S Supporting Information}

The Supporting Information is available free of charge on the ACS Publications website at DOI: 10.1021/acsomega.8b02875.
${ }^{1} \mathrm{H}$ NMR spectra of unmodified and modified chitosans; $\zeta$-potentials and hydrodynamic sizes of unmodified chitosans of different molecular weights; nomenclature used for the description of the modified chitosans; loading capacities and entrapment efficiencies of siRNA in the chitosan-based NPs; verification of the complexation by gel electrophoresis; colloidal stability of the nanocomplexes at different $\mathrm{pH}$ values; cell viabilities and relative cell survivals of the different chitosan-based NPs; two-dimensional and 3D confocal images for cellular uptake testing in RAW 264.7 cells (PDF)

\section{AUTHOR INFORMATION}

\section{Corresponding Authors}

*E-mail: eva.mailbox1@gmail.com (E.V.-A.).

*E-mail: silvia.barbosa@usc.es (S.B.).

ORCID 우

Silvia Barbosa: 0000-0003-1831-1686

Pablo Taboada: 0000-0002-2903-7857

Author Contributions

${ }^{\S}$ E.V.-A. and B.H.L. contributed equally to this work.

Notes

The authors declare no competing financial interest.

\section{ACKNOWLEDGMENTS}

This work was supported by the Agencia Estatal de Investigación (AEI) through Project MAT2016-80266-R and Xunta de Galicia (Grupo de Referencia Competitiva ED431C 2018/26; Agrupación Estratégica en Materiales-AEMAT ED431E 2018/08). FEDER funds are also acknowledged. A.C. also thanks Xunta de Galicia for her postdoctoral fellowship. E.V.-A. and A.P. are also grateful to the Spanish Ministerio de Economia y Competitividad for their FPU fellowships. The authors also thank Instituto de Ortopedia y Tejidos Musculo-Esqueléticos for the facilities for performing the in vitro studies.

\section{REFERENCES}

(1) Morris, K. V.; Chan, S. W.-L.; Jacobsen, S. E.; Looney, D. J. Small Interfering RNA-Induced Transcriptional Gene Silencing in Human Cells. Science 2004, 305, 1289-1292.

(2) Elbashir, S. M.; Harborth, J.; Lendeckel, W.; Yalcin, A.; Weber, K.; Tuschl, T. Duplexes of 21-Nucleotide RNAs Mediate RNA Interference in Cultured Mammalian Cells. Nature 2001, 411, 494498.

(3) Dykxhoorn, D. M.; Novina, C. D.; Sharp, P. A. Killing the Messenger: Short RNAs that Silence Gene Expression. Nat. Rev. Mol. Cell Biol. 2003, 4, 457-467.

(4) Leuschner, P. J. F.; Ameres, S. L.; Kueng, S.; Martinez, J. Cleavage of the siRNA Passenger Strand during RISC Assembly in Human Cells. EMBO Rep. 2006, 7, 314-320.

(5) Heidel, J. D.; Hu, S.; Liu, X. F.; Triche, T. J.; Davis, M. E. Lack of Interferon Response in Animals to Naked siRNAs. Nat. Biotechnol. 2004, 22, 1579-1582.

(6) Sioud, M. On the Delivery of Small Interfering RNAs into Mammalian Cells. Expert Opin. Drug Delivery 2005, 2, 639-651.

(7) Zhang, S.; Zhao, B.; Jiang, H.; Wang, B.; Ma, B. Cationic Lipids and Polymers Mediated Vectors for Delivery of siRNA. J. Controlled Release 2007, 123, 1-10.

(8) Kumar, L. D.; Clarke, A. R. Gene Manipulation Through the Use of Small Interfering RNA (siRNA): From In Vitro to In Vivo Applications. Adv. Drug Delivery Rev. 2007, 59, 87-100.

(9) Liu, X.; Howard, K. A.; Dong, M.; Andersen, M. O.; Rahbek, U. L.; Johnsen, M. G.; Hansen, O. C.; Besenbacher, F.; Kjems, J. The 
Influence of Polymeric Properties on Chitosan/siRNA Nanoparticle Formulation and Gene Silencing. Biomaterials 2007, 28, 1280-1288.

(10) Katas, H.; Alpar, H. O. Development and Characterisation of Chitosan NPs for siRNA Delivery. J. Controlled Release 2006, 115, 216-225.

(11) Howard, K. A.; Rahbek, U. L.; Liu, X. D.; Damgaard, C. K.; Glud, S. Z.; Andersen, M. O.; Hovgaard, M. B.; Schmitz, A.; Nyengaard, J. R.; Besenbacher, F.; Kjems. RNA Interference In Vitro and In Vivo Using a Chitosan/siRNA Nanoparticle System. Mol. Ther. 2006, 14, 476-484.

(12) Li, L.; Hu, X.; Zhang, M.; Ma, S.; Yu, F.; Zhao, S.; Liu, N.; Wang, Z.; Wang, Y.; Guan, H.; Pan, X.; Gao, Y.; Zhang, Y.; Liu, Y.; Yang, Y.; Tang, X.; Li, M.; Liu, C.; Li, Z.; Mei, X. Dual TumorTargeting Nanocarrier System for siRNA Delivery Based on pRNA and Modified Chitosan. Mol. Ther.-Nucleic Acids 2017, 8, 169-183. (13) Mao, S.; Sun, W.; Kissel, T. Chitosan-based Formulations for Delivery of DNA and siRNA. Adv. Drug Delivery Rev. 2010, 62, 1227.

(14) Ragelle, H.; Vandermeulen, G.; Préat, V. Chitosan-based siRNA Delivery Systems. J. Controlled Release 2013, 172, 207-218.

(15) Zhang, J.; He, C.; Tang, C.; Yin, C. Ternary Polymeric Nanoparticles for Oral siRNA Delivery. Pharm. Res. 2013, 30, 12281239.

(16) Varkouhi, A. K.; Verheul, R. J.; Schiffelers, R. M.; Lammers, T.; Storm, G.; Hennink, W. E. Gene Silencing Activity of siRNA Polyplexes Based on Thiolated N,N,N-trimethylated Chitosan. Bioconjugate Chem. 2010, 21, 2339-2346.

(17) Lee, S. J.; Huh, M. S.; Lee, S. Y.; Min, S.; Lee, S.; Koo, H.; Chu, J. U.; Lee, K. E.; Jeon, H.; Choi, Y.; Choi, K.; Byun, Y.; Jeong, S. Y.; Park, K.; Kim, K.; Kwon, I. C. Tumor-homing Poly-siRNA/glycol Chitosan Self-cross-linked Nanoparticles for Systemic siRNA Delivery in Cancer Treatment. Angew. Chem., Int. Ed. 2012, 51, 7203-7207.

(18) Malhotra, M.; Tomaro-Duchesneau, C.; Prakash, S. Synthesis of TAT Peptide-tagged PEGylated Chitosan Nanoparticles for siRNA Delivery Targeting Neurodegenerative Diseases. Biomaterials 2013, 34, 1270-1280.

(19) Guţoaia, A.; Schuster, L.; Margutti, S.; Laufer, S.; Schlosshauer, B.; Krastev, R.; Stoll, D.; Hartmann, H. Fine-tuned PEGylation of Chitosan to Maintain Optimal siRNA-nanoplex Bioactivity. Carbohydr. Polym. 2016, 143, 25-34.

(20) Luo, Y.; Zhai, X.; Ma, C.; Sun, P.; Fu, Z.; Liu, W.; Xu, J. An Inhalable Beta(2)- Adrenoceptor Ligand-directed Guanidinylated Chitosan Carrier for Targeted Delivery of siRNA to Lung. J. Controlled Release 2012, 162, 28-36.

(21) Liu, Z.; Zhang, Z.; Zhou, C.; Jiao, Y. Hydrophobic Modifications of Cationic Polymers for Gene Delivery. Prog. Polym. Sci. 2010, 35, 1144-1162.

(22) Liu, W. G.; Zhang, X.; Sun, S. J.; Sun, G. J.; Yao, K. D.; Liang, D. C.; Guo, G.; Zhang, J. Y. N-alkylated Chitosan as a Potential Nonviral Vector for Gene Transfection. Bioconjugate Chem. 2003, 14, $782-789$.

(23) Kuhn, P. S.; Levin, Y.; Barbosa, M. C. Charge Inversion in DNA-Amphiphile Complexes: Possible Application to Gene Therapy. Phys. A 1999, 274, 8-18.

(24) Douglas, K. L.; Piccirillo, C. A.; Tabrizian, M. Cell Linedependent Internalization Pathways and Intracellular Trafficking Determine Transfection Efficiency of Nanoparticle Vectors. Eur. J. Pharm. Biopharm. 2008, 68, 676-687.

(25) Robles, E.; Villar, E.; Alatorre-Meda, M.; Burboa, M. G.; Valdez, M. A.; Taboada, P.; Mosquera, V. Effects of the Hydrophobization on Chitosan-Insulin Nanoparticles Obtained by an Alkylation Reaction on Chitosan. J. Appl. Polym. Sci. 2013, 129, 822-834.

(26) Yalpani, M.; Hall, L. D. Some Chemical and Analytical Aspects of Polysaccharide Modifications. III. Formation of Branched-Chain, Soluble Chitosan Derivatives. Macromolecules 1984, 17, 272-281.

(27) Desbrières, J.; Martinez, C.; Rinaudo, M. Hydrophobic derivatives of Chitosan: Characterization and Rheological Behavior. Int. J. Biol. Macromol. 1996, 19, 21-28.
(28) Robles, E.; Juárez, J.; Burboa, M.; Gutiérrez, L.; Taboada, P.; Mosquera, V.; Valdez, M. Properties of Insulin-Chitosan Complexes Obtained by an Alkylation Reaction on Chitosan. J. Appl. Polym. Sci. 2014, 131, No. 39999.

(29) Fernández-Urrusuno, R.; Calvo, P.; Remuñán-López, C.; VilaJato, J. L.; Alonso, M. J. Enhancement of Nasal Absorption of Insulin Using Chitosan Nanoparticles. Pharm. Res. 1999, 16, 1576-1581.

(30) Provencher, S. W.; Stepanek, P. Global Abalysis if Dynamic Light Scattering Autocorrelation Functions. Part. Part. Syst. Charact. 1996, 13, 291-294.

(31) Alatorre-Meda, M.; Taboada, P.; Krajewska, B.; Willemeit, M.; Deml, A.; Klösel, R.; Rodríguez, J. R. DNA-Poly(diallyldimethylammonium chloride) Complexation and Transfection Efficiency. J. Phys. Chem. B 2010, 114, 9356-9366.

(32) Alatorre-Meda, M.; Taboada, P.; Hartl, F.; Wagner, T.; Freis, M.; Rodríguez, J. R. The Influence of Chitosan Valence on the Complexation and Transfection of DNA: The Weaker the DNAchitosan Binding the Higher the Transfection Efficiency. Colloids Surf., B 2011, 82, 54-62.

(33) Lv, H.-S.; Liu, J.; Zhao, J.; Zhao, B.-X.; Miao, J.-Y. Highly Selective and Sensitive pH-Responsive Fluorescent Probe in Living HeLa and HUVEC Cells. Sens. Actuators, B 2013, 177, 956-963.

(34) Dou, J.; Zhang, H.; Liu, X.; Zhang, M.; Zhai, G. Preparation and Evaluation In Vitro and In Vivo of Docetaxel-loaded Mixed Micelles for Oral Administration. Colloids Surf., B 2014, 114, 20-27.

(35) Chen, M.; Gao, S.; Dong, M.; Song, J.; Yang, C.; Howard, K. A.; Kjems, J.; Besenbacher, F. Chitosan/siRNA Nanoparticles Encapsulated in PLGA Nanofibers for siRNA Delivery. ACS Nano 2012, 6, 4835-4844.

(36) Malhotra, M.; Tomaro-Duchesneau, C.; Saha, S.; Kahouli, I.; Prakash, S. Development and Characterization of Chitosan-PEG-TAT Nanoparticles for the Intracellular Delivery of siRNA. Int. J. Nanomed. 2013, 8, 2041-2052.

(37) Jean, M.; Alameh, M.; De Jesus, D.; Thibault, M.; Lavertu, M.; Darras, V.; Nelea, M.; Buschmann, M. D.; Merzouki, A. ChitosanBased Therapeutic Nanoparticles for Combination Gene Therapy and Gene Silencing of In Vitro Cell Lines Relevant to Type 2 Diabetes. Eur. J. Pharm. Sci. 2012, 45, 138-149.

(38) Taboada, P.; Fernández, Y.; Mosquera, V. Interactions of Two Amphiphilic Penicillins with Myoglobin in Aqueous Buffered Solutions: A Thermodynamic and Spectroscopy Study. Biomacromolecules 2004, 5, 2201-2211.

(39) Castro, E.; Taboada, P.; Mosquera, V. Behavior of a Styrene Oxide-Ethylene Oxide Diblock Copolymer/Surfactant System: A Thermodynamic and Spectroscopy Study. J. Phys. Chem. B 2005, 109, 5592-5599.

(40) Dam, T. K.; Gerken, T. A.; Cavada, B. S.; Nascimento, K. S.; Moura, T. R.; Brewer, C. F. Binding Studies of $\alpha$-GalNAc-Specific Lectins to the $\alpha$-GalNAc (Tn-Antigen) Form of Porcine Submaxillary Mucin and its Smaller Fragments. J. Biol. Chem. 2007, 282, 2825628263.

(41) Zhang, J.; Chen, X. G.; Li, Y.; Liu, C. S. Self-Assembled Nanoparticles Based on Hydrophobically Modified Chitosan as Carriers for Doxorubicin. Nanomedicine 2007, 3, 258-265.

(42) Sonia, T. A.; Rekha, M. R.; Sharma, C. P. Bioadhesive Hydrophobic Chitosan Microparticles for Oral Delivery of Insulin: In Vitro Characterization and In vivo Uptake Studies. J. Appl. Polym. Sci. 2011, 119, 2902-2910.

(43) Alatorre-Meda, M.; Taboada, P.; Sabín, J.; Krajewska, B.; Varela, L. M.; Rodríguez, J. R. DNA-Chitosan Complexation: A Dynamic Light Scattering Study. Colloids Surf., A 2009, 339, 145152.

(44) Elsayed, A.; Al-Remawi, M.; Farouk, A.; Badwan, A. InsulinChitosan Polyelectrolyte Nanocomplexes: Preparation, Characterization and Stabilization of Insulin. Sudan J. Med. Sci. 2010, 5, 99110 .

(45) Sadeghi, A. M.; Dorkoosh, F. A.; Avadi, M. R.; Saadat, P.; Rafiee-Tehrani, M.; Junginger, H. E. Preparation, Characterization and Antibacterial Activities of Chitosan, $\mathrm{N}$-trimethyl Chitosan 
(TMC) and N-diethylmethyl Chitosan (DEMC) Nanoparticles Loaded with Insulin Using Both the Ionotropic Gelation and Polyelectrolyte Complexation Methods. Int. J. Pharm. 2008, 355, 299-306.

(46) Al-Qadi, S.; Alatorre-Meda, M.; Martin-Pastor, M.; Taboada, P.; Remunán-López, C. the role of Hyaluronic Acid Inclusion on the energetics of Encapsulation and Release of a Protein Molecule from Chitosan-Based Nanoparticles. Colloids Surf., B 2016, 141, 223-232.

(47) Ma, Z.; Yeoh, H. H.; Lim, L.-Y. Formulation pH Modulates the Interaction of Insulin with Chitosan Nanoparticles. J. Pharm. Sci. 2002, 91, 1396-1404.

(48) Morris, G. A.; Castile, J.; Smith, A.; Adams, G. G.; Harding, S. E. The Effect of Prolonged Storage at Different Temperatures on the Particle Size Distribution of Tripolyphosphate (TPP)-Chitosan Nanoparticles. Carbohydr. Polym. 2011, 84, 1430-1434.

(49) Ehrenberg, M. S.; Friedman, A. E.; Finkelstrin, J. N.; Oberdörster, G.; McGrath, J. L. The Influence of Protein Adsorption on Nanoparticle Association with Cultured Endothelial Cells. Biomaterials 2009, 30, 603-610.

(50) Walczyk, D.; Bombelli, F. B.; Monopoli, M. P.; Lynch, I.; Dawson, K. A. What the Cell "Sees" in Bionanoscience. J. Am. Chem. Soc. 2010, 132, 5761-5768.

(51) Jeon, S. I.; Andrade, J. D. Protein-Surface Interactions in the Presence of Polyethylene Oxide: II. Effect of Protein Size. J. Colloid Interface Sci. 1991, 142, 159-166.

(52) Gessner, A.; Lieske, A.; Paulke, B. R.; Muller, R. H. Functional Groups on Polystyrene Model Nanoparticles: Influence on Protein Adsorption. J. Biomed. Mater. Res., Part A. 2003, 65, 319-326.

(53) Quong, D.; Neufeld, R. J. DNA Protection from Extracapsular Nucleases within Chitosan- or Poly-L-lysine-Coated Alginate Beads. Biotechnol. Bioeng. 1998, 60, 124-134.

(54) Braasch, D. A.; Jensen, S.; Liu, Y.; Kaur, K.; Arar, K.; White, M. A.; Corey, D. R. RNA Interference in Mammalian Cells by Chemically-Modified RNA. Biochemistry 2003, 42, 7967-7975.

(55) Jo, H. G.; Min, K. H.; Nam, T. H.; Na, S. J.; Park, J. H.; Jeong, S. Y. Prolonged Antidiabetic Effect of Zinc-crystallised Insulin Loaded Glycol Chitosan Nanoparticles in Type 1 Diabetic Rats. Arch. Pharm. Res. 2008, 31, 918-923.

(56) Raja, A. G.; Katas, H.; Wen, T. J. Stability, Intracellular Delivery, and Release of siRNA from Chitosan Nanoparticles Using Different Cross-Linkers. PLoS One 2015, 10, No. e0128963.

(57) Shen, J. W.; Li, J.; Zhao, Z.; Zhang, L.; Peng, G.; Liang, L. Molecular Dynamics Study on the Mechanism of Polynucleotide Encapsulation by Chitosan. Sci. Rep. 2017, 7, No. 5050.

(58) Kim, T.-H.; Jiang, H.-L.; Jere, D.; Park, I.-K.; Cho, M.-H.; Nah, J.-W.; Choi, Y.-J.; Akaike, T.; Cho, C.-S. Chemical Modification of Chitosan as A Gene Carrier In Vitro and In Vivo. Prog. Polym. Sci. 2007, 32, 726-753.

(59) Yang, H.-C.; Hon, M.-H. The Effect of the Molecular Weight of Chitosan Nanoparticles and Its Application on Drug Delivery. Microchem. J. 2009, 92, 87-91.

(60) Hou, Y.; Hu, J.; Park, H.; Lee, M. Chitosan Based Nanoparticles as a Sustained Protein Release Carrier for Tissue Engineering Applications. J. Biomed. Mater. Res. A 2012, 100, 939947.

(61) Li, F.; Liu, W. G.; De Yao, K. Preparation of Oxidized GlucoseCrosslinked N-Alkylated Chitosan Membrane and In Vitro Studies of pH-Sensitive Drug Delivery Behaviour. Biomaterials 2002, 23, 343347.

(62) Kagan, V. E.; Tyurina, Y. Y.; Tyurin, V. A.; Konduru, N. V.; Potapovich, A. I.; Osipov, A. N.; Kisin, E. R.; Schwegler-Berry, D.; Mercer, R.; Castranova, V.; Shvedova, A. A. Direct and Indirect Effects of Single Walled Carbon Nanotubes on RAW 264.7 Macrophages: Role of Iron. Toxicol. Lett. 2006, 165, 88-100.

(63) Xia, T.; Kovochich, M.; Liong, M.; Mädler, L.; Gilbert, B.; Shi, H.; Yeh, J. I.; Zink, J. I.; Nel, A. E. Comparison of the Mechanism of Toxicity of Zinc Oxide and Cerium Oxide Nanoparticles Based on Dissolution and Oxidative Stress Properties. ACS Nano 2008, 2, 2121-2134.
(64) Shukla, R.; Bansal, V.; Chaudhary, M.; Basu, A.; Bhonde, R. R.; Sastry, M. Biocompatibility of Gold Nanoparticles and Their Endocytotic Fate Inside the Cellular Compartment: A Microscopic Overview. Langmuir 2005, 21, 10644-10654.

(65) Asthana, G. S.; Asthana, A.; Kohli, D. V.; Vyas, S. P. Mannosylated Chitosan Nanoparticles for Delivery of Antisense Oligonucleotides for Macrophage Targeting. BioMed. Res. Int. 2014, 2014, 1-17.

(66) Moghimi, S. M.; Symonds, P.; Murray, J. C.; Hunter, A. C.; Debska, G.; Szewczyk, A. A Two-Stage Poly(Ethylenimine)-Mediated Cytotoxicity: Implications for Gene Transfer/Therapy. Mol. Ther. 2005, 11, 990-995.

(67) Gao, S.; Chen, J.; Dong, L.; Ding, Z.; Yang, Y.; Zhang, J. Targeting Delivery of Oligonucleotide and Plasmid DNA to Hepatocyte via Galactosylated Chitosan Vector. Eur. J. Pharm. Biopharm. 2005, 60, 327-334.

(68) Sajid, M.; Ilyas, M.; Basheer, C.; Tariq, M.; Daud, M.; Baig, N.; Shehzad, F. Impact of nanoparticles on human and environment: review of toxicity factors, exposures, control strategies, and future prospects. Environ. Sci. Pollut. Res. 2015, 22, 4122-4143.

(69) Fröhlich, E. The Role of Surface Charge in Cellular Uptake and Cytotoxicity of Medical Nanoparticles. Int. J. Nanomed. 2012, 7 , 5577-5591.

(70) Lameijer, M. A.; Tang, J.; Nahrendorf, M.; Beelen, R. H. J.; Mulder, W. J. M. Monocytes and Macrophages as Nanomedicinal Targets for Improved Diagnosis and Treatment of Disease. Expert Rev. Mol. Diagn. 2013, 13, 567-580.

(71) Kim, Y.-K.; Jiang, H.-L.; Guo, D.-D.; Choi, Y.-J.; Cho, M.-H.; Akaike, T.; Cho, C.-S. In Chitosan-Based Systems for Biopharmaceuticals: Delivery, Targeting and Polymer Therapeutics; Sarmento, B., das Neves, J., Eds.; Wiley \& Sons, 2012; Chapter 15, pp 255-273.

(72) Kurisawa, M.; Yokoyama, M.; Okano, T. Transfection Efficiency Increases by Incorporating Hydrophobic Monomer Units into Polymeric Gene Carriers. J. Controlled Release 2000, 68, 1-8.

(73) Chae, S. Y.; Son, S.; Lee, M.; Jang, M. K.; Nah, J. W. Deoxycholic Acid Conjugated Chitosan Oligosaccharide Nanoparticles for Efficient Gene Carrier. J. Controlled Release 2005, 109, 330-344.

(74) Hu, F.-Q.; Zhao, M.-D.; Yuan, H.; You, J.; Du, Y.-Z.; Zeng, S. Novel Chitosan Oligosaccharide-Stearic Acid Micelles for Gene Delivery: Properties and In Vitro Transfection Studies. Int. J. Pharm. 2006, 315, 158-166.

(75) Nouri, F.; Sadeghpour, H.; Heidari, R.; Dehshahri, A. Preparation, Characterization, and Transfection Efficiency of Low Molecular Weight Polyethylenimine-Based Nanoparticles for Delivery of the Plasmid Encoding CD200 Gene. Int. J. Nanomed. 2017, 12, $5557-5569$

(76) Techaarpornkul, S.; Wongkupasert, S.; Opanasopit, P.; Apirakaramwong, A.; Nunthanid, J.; Ruktanonchai, U. ChitosanMediated siRNA Delivery In Vitro: Effect of Polymer Molecular Weight, Concentration and Salt Forms. AAPS PharmSciTech 2010, 11, $64-72$. 